\title{
A review on the impact of humidity during electrospinning: from the nanofiber structure engineering to the applications
}

\author{
Domitille Mailley, Anne Hébraud, and Guy Schlatter
}

Dr. D. Mailley, Dr. A. Hébraud, Prof. G. Schlatter

Institut de Chimie et Procédés pour l'Energie, l'Environnement et la Santé, ICPEES UMR 7515, Université de Strasbourg, Ecole Européenne de Chimie, Polymères et Matériaux, CNRS, 25 rue Becquerel, 67087 Strasbourg Cedex 2, France

Email: guy.schlatter@unistra.fr

Keywords: electrospinning, nanofibers, non-woven mats, humidity, needleless, electrospinning.

\begin{abstract}
Electrospinning is the process of choice for the elaboration of nanofibrous mats. During the process, a thin and continuous charged jet of a polymer solution is travelling from an emitter subjected to a high voltage towards a grounded collector. Although the duration of the jet travel is in the order of few tens of milliseconds, the physical interactions acting between the jet and the air play a key role on the resulting fiber morphology. These interactions mainly rely on the amount of water molecules in air. This review deals with the effect of humidity during electrospinning on solvent evaporation, the solidification rate of nanofibers and finally, on the morphology at length scales ranging from the non-woven mat, the nanofiber itself down to the polymer crystal. Original electrospinning processes operating under specific environmental conditions as well as the specificities encountered in needleless and free-surface electrospinning dedicated to industrial scale mass production are also discussed. Then, it is shown how the control of humidity during electrospinning and the understanding of its influence on the fibrous structure can be exploited to target various applications dedicated to energy, environment and health. Finally, current challenges and ideas for future research and new developments are presented.
\end{abstract}




\section{Introduction}

Electrospinning ${ }^{[1-4]}$ is a process allowing the production of a nanofibrous nonwoven mat from a polymer solution subjected to the action of a high difference of electric potential between an emitter and a grounded collector located at a distance of 10 to $30 \mathrm{~cm}$ from the emitter. The polymer solution is placed in a syringe, pushed out of the syringe at constant rate towards a metallic emitter (a needle at the lab scale). When the electric field established between the needle and the collector is high enough (i.e. in the order of 1 $\mathrm{kV} / \mathrm{cm}$ ), the droplet of polymer solution exiting the needle takes the shape of the so-called Taylor cone from which a charged straight jet having a length of several $\mathrm{mm}$ is propelled. Then, the jet is subjected to unstable whipping movements due to the presence of surface charges. These whipping movements play a key role as they ensure the efficient evaporation of the solvent by convection. Thereby the jet elongates and solidifies. Eventually a dry fiber with an average diameter ranging from tens of nm to few microns is deposited on the collector in the form of a nonwoven mat. The final structure of the mat depends on the processing parameters, the polymer solution properties and ambient parameters such as the temperature and humidity. ${ }^{[5-8]}$

In a practical point of view, humidity of ambient air is assessed with the absolute humidity $(\mathrm{AH})$ which is either defined by the number of moles of water per total moles of air or by the mass of water for a given mass of dry air. ${ }^{[9]} \mathrm{AH}$ is a thermodynamic parameter independent of any other physical parameters. The dew point $\left(\mathrm{T}_{\mathrm{dp}}\right)$ is another used parameter directly related to $\mathrm{AH}$ corresponding to the temperature at which water vapor starts to condensate and forms the first liquid droplet. Unfortunately, the direct 
measurement of $\mathrm{AH}$ as well as $\mathrm{T}_{\mathrm{dp}}$ is not possible and humidity of ambient air is, instead, evaluated through the relative humidity $(\mathrm{RH})$ which depends on the pressure and the temperature $\mathrm{T}$ of air. The links between $\mathrm{AH}, \mathrm{T}_{\mathrm{dp}}, \mathrm{RH}$ and the temperature are clarified in Figure 1. RH represents, in percent, the ratio between the partial pressure of water vapor and the equilibrium vapor pressure of water at the same temperature.

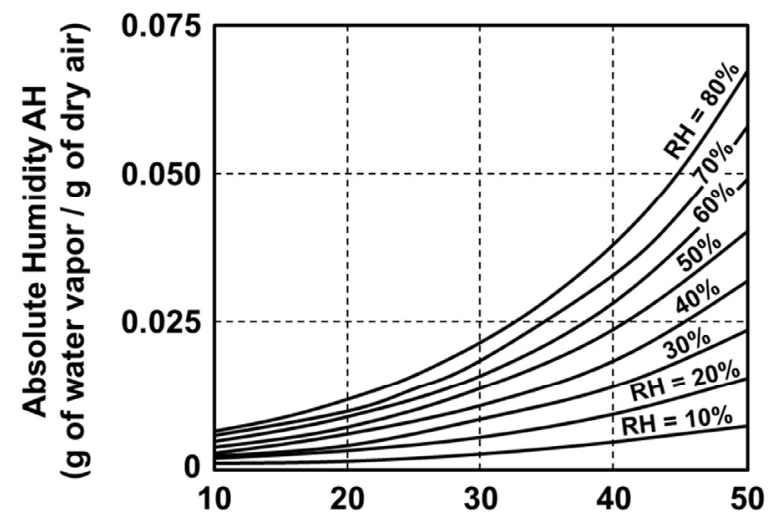

a)

Temperature of air $\mathrm{T}\left({ }^{\circ} \mathrm{C}\right)$

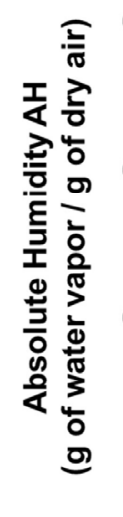

b)

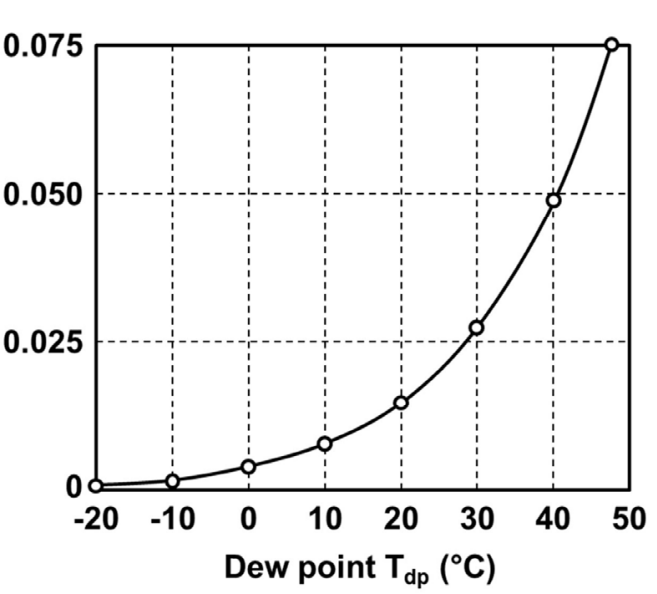

Figure 1: a) Absolute humidity at sea-level atmospheric pressure as a function of relative humidity and temperature. Reproduced with permission. ${ }^{[9]}$ Copyright 2012, Elsevier. b) Absolute humidity at sea-level atmospheric pressure as a function of the dew point calculated from. ${ }^{[10]}$

The effect of the structure at the scales of the nanofibers and the mats on the functional properties of the resulting materials dedicated to targeted applications is of prime importance. $^{[1,11,12]}$ For example, the fiber diameter was found to be a predominant parameter for electrospun vascular grafts ${ }^{[13]}$ and liquid filtration. ${ }^{[14]}$ Beaded fibers have been studied for photonic applications, ${ }^{[15]}$ controlled release applications ${ }^{[16,17]}$ and fog harvesting applications. ${ }^{[18]}$ Porous fibers are of great interest for absorption applications such as oil, ${ }^{[19]}$ phenol and iodine. ${ }^{[20]}$ Cotton-like structures can improve cell adhesion ${ }^{[21]}$ and cell penetration inside electrospun scaffolds for biomedical applications. ${ }^{[22,23]}$ 
Oriented fibers have been used to enhance liquid filtration efficiencies ${ }^{[24]}$ and for tendon and ligament tissue engineering. ${ }^{[25]}$ Finally, mats containing nanonets are being developed for state-of-the-art sensing and filtering applications. ${ }^{[26]}$ Thus, understanding the effect of humidity on the structure of fibers and mats is mandatory to target efficiently the functional properties of the fabricated material.

Contrary to all the other parameters, the effect of humidity during electrospinning on the nanofiber morphology and properties has scarcely been studied and to our knowledge, only one review focusing on this subject has been yet published. ${ }^{[27]}$ In the present review, the objective is to show that humidity is a key parameter during the fabrication of fibrous membranes by electrospinning. First, we will clarify the role of humidity on the solvent evaporation and fiber solidification rates during the processing. Specificities encountered in needleless and free-surface electrospinning are also discussed. Then, we aim at indexing and explaining the effect of humidity on the morphology of the fibrous material at the different length scales: (i) at the scale of the individual nanofiber and (ii) at the scale of the fibrous mat. Original electrospinning processes in which specific air environment can be set in order to produce well-structured fibrous membranes will then be presented and discussed. In the end, relevant examples for which the precise control of humidity during electrospinning is of paramount importance to develop dedicated fibrous structures adapted for applications in various fields such as health, environment and energy will be presented. 


\section{Role of humidity during electrospinning on solvent evaporation and fiber solidification}

\subsection{Effect of humidity on solvent evaporation}

In the electrospinning process, solvent evaporation occurs at the tip of the needle and during the jet flight until it reaches the collector. The polymer solution crosses three domains: i) the Taylor cone, ii) the straight jet and iii) the bending/whipping region (see Figure 2a). Because the relative velocity of air with the surface of the polymer solution is low in the Taylor cone and the straight jet as well, solvent evaporation occurs only by a diffusion mechanism within these two regions. However, in the bending region, evaporation happens along the electrospun jet in few tens of milliseconds by a convection mechanism due to the whipping movements. ${ }^{[28,29]}$ These whipping movements elongate the jet, generate more surface and lead to high air velocities both tangentially and transversally to the jet axis promoting thus an efficient solvent evaporation from the surface of the jet. This step of evaporation is the most important because it leads to the deposition of a dry nanofiber on the collector. The solvent evaporation rate $\dot{m}_{\text {evap }}$ of a short segment of jet of length $d \xi$ is linked with the variation of the jet flow rate $Q$ through the mass balance:

$$
\dot{m}_{\text {evap }}=-\rho d Q
$$

Where $\rho$ is the solution density. The direct link between the evaporation rate $\dot{m}_{\text {evap }}$ and humidity during the electrospinning process is clarified in the following equation. ${ }^{[30]}$

$$
\dot{m}_{\text {evap }}=-\rho h_{s}\left[c_{s, e q}(T)-c_{s \infty}\right] 2 \pi R d \xi
$$

Where $h_{s}$ is the mass transfer coefficient of solvent, $c_{s, e q}(T)$ the saturation vapor concentration of solvent at temperature $\mathrm{T}, c_{s \infty}$ the vapor concentration in the atmosphere far from the jet, $\xi$ the arc length of the jet and $R$ the cross-sectional radius of the jet at $\xi$. 
In the case when pure water is used as solvent and assuming that air and vapor are ideal gas, $\left[c_{s, e q}(T)-c_{s \infty}\right]$ is proportional to $\left[1-\frac{R H}{100}\right]$. Thus, under these assumptions and using an empiric expression of the mass transfer coefficient of water, Cai and Gevelber ${ }^{[31]}$ proposed the following expression of $\dot{m}_{\text {evap }}$ :

$$
\dot{m}_{\text {evap }}=1.95 v_{a}^{1 / 6} D_{a}^{1 / 2} \rho V_{\text {air }}^{1 / 3} R^{1 / 3}\left[1-\frac{R H}{100}\right] d \xi
$$

Where:

$v_{a}:$ Kinematic viscosity of air

$D_{a}:$ Water vapor diffusion rate in air

$V_{\text {air }}$ : Relative velocity of air at the jet surface

Thus, the evaporation rate depends on the type of solvent: i.e. aqueous or non-aqueous solvents. When the solvent is not aqueous, then $c_{s \infty}$ is equal to 0 leading to evaporation, which only depends on the saturated vapor pressure of the solvent. However, in the case of aqueous solvents, the evaporation rate depends on water vapor concentration in the environment. When $c_{s \infty}$ is close to the saturation vapor concentration at temperature $\mathrm{T}$ (i.e. when the difference $c_{s, e q}(T)-c_{s \infty}$ in eq. 1 is close to 0 ), only little water can be evaporated from the jet, before the equilibrium is reached. In that case, the evaporation of the jet is slow. On the contrary, when the water vapor concentration in air is far below the saturated vapor concentration, the evaporation of the water contained in the electrospun jet is facilitated. ${ }^{[32,33]}$

Humidity also affects indirectly the evaporation rate as it can influence the removal of charges from the surface of the jet. ${ }^{[34,35]}$ Although air is commonly considered as an insulating gas, under high electric potentials it becomes able to conduct a leakage current 
thanks to the formation of ions in the air. ${ }^{[36]}$ As a result, for any kind of solvent (Figure 2a), the electrospinning jet gets partially discharged through recombination with the surrounding ionized air. With an increase in humidity, the ionization of the air is enhanced due to the higher amount of water vapor molecules in the air. Water vapor molecules can be easily ionized thanks to their low ionization energy compared to other species present in the air such as nitrogen. ${ }^{[37]}$ Thus, at high humidity, the electrospinning jet is discharged even more. As the charge density on the jet is lowered at high humidity, the amplitude of the whipping movements is reduced, which slows down the evaporation rate of the solvent. $^{[38-41]}$

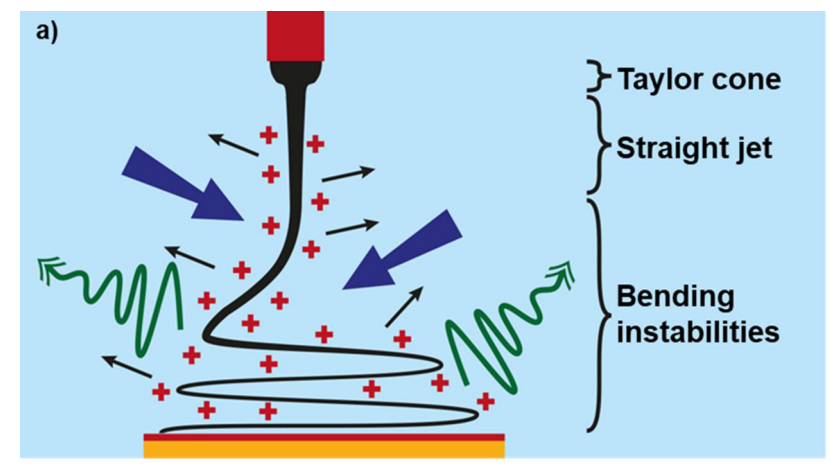

High RH for all solvents (except the aqueous): Charge dissipation: 2 competitive effects

$\rightarrow$ Smaller whipping movements (leading to coarser fibers)

$\rightarrow$ Slower evaporation (leading to smaller fiber)

High RH in the case of aqueous solvents:

Water absorption

$\rightarrow$ Slower evaporation (leading to coarser fibers)

+ Charge

$\longrightarrow$ Flux of charges

$\mu^{-7}$ Solvent or

$\rightarrow$ Water absorption

Water vapor

b) Hydrophilic polymers

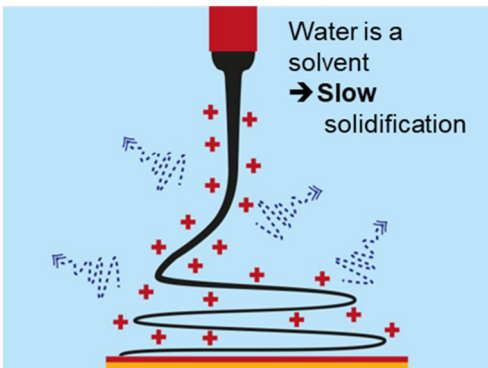

c) Hygroscopic polymers

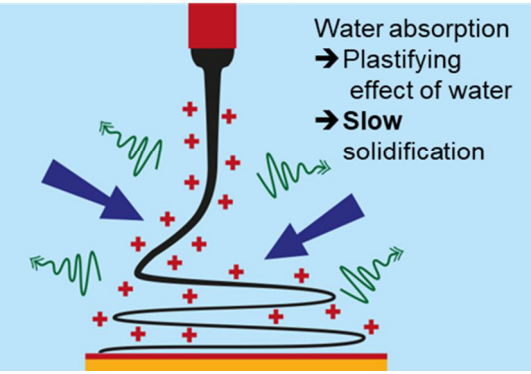

d) Hydrophobic polymers

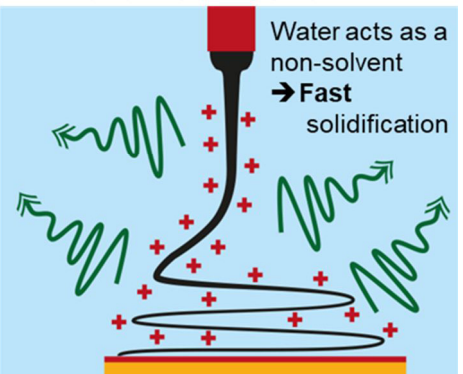

Figure 2. a) Effects of humidity at high RH on the evaporation rate of the electrospinning jet as a function of the solvent. Effects of humidity at high RH on the solidification rate of the electrospinning jet as a function of the polymer hidrophilicity: b) hydrophilic, c) hygroscopic and d) hydrophobic. 


\subsection{Effect of humidity on fiber solidification}

Electrospun fibers can solidify thanks to phase separations during the electrospinning process. Phase separation happens when the solution composition crosses the miscibility limit which generates polymer and solvent rich regions. ${ }^{[42]}$ Phase diagrams can be used to predict the miscibility limit of a solution and consequently phase separation. ${ }^{[9,43,44]}$ Two mechanisms can take place during phase separation in electrospinning: thermal induced phase separation (TIPS) and vapor induced phase separation (VIPS). ${ }^{[45-47]}$ TIPS is a phase separation mechanism induced by the rapid cooling of a homogeneous polymer solution. Thus, in electrospinning, TIPS may happen when, because of the evaporation of the solvent, the surface of the fibers cools down. In that case, the limit of solubility is lowered and the solution may precipitate. During VIPS, the vapor acts as a non-solvent. This leads to the precipitation of the polymer out of the solution. After fiber solidification, dried fibers are deposited on the collector and form the nonwoven mat. Humidity can influence the solidification rate of the fibers and favor one of the phase separations mechanisms. Its effect varies along with the nature of the polymer and the solvent.

It has been previously seen that humidity postpones the evaporation of the solvent if the solvent is water or if the solvent is hydrophilic. Thus, it delays the solidification of fibers (Figure 2b). ${ }^{[32,33,48]}$ Hygroscopic polymers can also absorb a small amount of water present in the air. As a result, a tremendous reduction of the glass transition due to a plasticizing effect is observed which could thus favor the elongation of the jet during longer time before final solidification. ${ }^{[49-51]}$ As a result, fibers can elongate during longer times before solidifying. So, high humidity, which promotes the absorption of water, postpones solidification and enables electrospun jets to stretch more out (Figure 2c). ${ }^{[48,52,53]}$ When a hydrophobic polymer is used, water vapor acts as a non-solvent for the 
polymer. Consequently, the increase of humidity leads to a faster solidification rate of the fibers (Figure 2d). ${ }^{[38,48,54]}$

\subsection{Specificities in case of needleless electrospinning for industrial production rate}

Nowadays, electrospinning is a mature process allowing the production of fibrous mats at an industrial scale thanks to the use of dedicated needleless emitters. Needleless electrospinning allows the simultaneous emission of jets using specifically designed emitters which can be classified in two main groups: ${ }^{[55]}$ (i) stationary emitters such as bubbles, ${ }^{[56]}$ cleft $^{[57]}$ mushroom ${ }^{[58]}$ or Elmarco's wire spinnerets and (ii) non-stationary rotary emitters such as cylinder, ${ }^{[59]}$ disc $^{[60]}$ or rotary bead wires. ${ }^{[61]}$ Each of these emitters show advantages/disadvantages in terms of production rate, cost, simplicity of maintenance, cleaning and distribution of the fiber diameter. ${ }^{[55,62]}$ Among these aspects, it is worth noting that the interactions between ambient air and the polymer solution in the vicinity of the jet emission zone are highly dependent on the type of needleless electrospinning system and are obviously very different from those observed for standard needle electrospinning. Indeed, a high surface area is generally exposed to ambient air increasing thus the effect of humidity around the Taylor cones as well as on the freesurface of the emitter between two consecutive Taylor cones. Such interactions may favor an early polymer precipitation in case of hydrophobic polymer solubilized in organic processed at high $\mathrm{RH}$ or an early evaporation and even solidification in the case of aqueous solution of hydrophilic polymers processed at low RH. Moreover, many of these needleless free surfaces emitters are subjected to exchanges with air along much larger residence times than what it is generally experienced in the case of needle electrospinning 
favoring the increase of the solution viscosity and the polymer precipitation. ${ }^{[62]}$ These detrimental effects can even be amplified in the case of non-stationary rotary emitters due to air convection at the emitter's free-surfaces. However, the use of an automatic cleaningdelivery system may prevent such effects by cleaning the emitter's free surface and supplying new fresh solution.

\section{Effect of humidity at the scale of the fiber}

As a consequence of the effect of the humidity on the electrospinning process (amount of charges, evaporation, solidification) the final morphology of fibers is affected as summarized in Table 1. Experiments have shown that the average diameter of the fibers may be influenced by humidity as well as the external shape of the fibers (beads-on-string fibers, beads) and the internal morphology of the fiber (surface roughness, pores located in the fiber shell or in the whole volume of the fiber) depending on whether a hydrophilic or a hydrophobic polymer is used. 
Table 1. Effects of RH on the morphology of the fibers regarding various polymer/solvent electrospinning systems. For comparison reasons, the $\mathrm{RH}$ values were taken from the literature and recalculated at a reference temperature of $\mathrm{T}_{\text {ref }}=20^{\circ} \mathrm{C}$. Fiber diameters are mentioned between brackets in the case of porous fibers.

\begin{tabular}{|c|c|c|c|c|c|}
\hline Ref & Polymer & Solvent & $\mathbf{T}\left({ }^{\circ} \mathbf{C}\right)$ & RH (\%) & Effects \\
\hline [9] & PS & DMF & 20 & $5-10$ & Bead-on-string fibers \\
\hline$[33]$ & PEO & Water & 22 & $\geq 53$ & Bead-on-string fibers \\
\hline [63] & $\begin{array}{l}\text { Gelatin- } \\
\mathrm{NaCl}\end{array}$ & Formic acid & 24 & 60 & Bead-on-string fibers \\
\hline$[32]$ & PVA & Water & 24 & 70 & Beads \\
\hline$[32]$ & PEO & Acetic acid & 24 & 50 & Beads \\
\hline$[38]$ & PAN & DMF & 22 & $\geq 20$ & Rough surface \\
\hline$[64]$ & PS & THF/DMF $1 / 4$ & 24 & 20 & Rough surface \\
\hline [39] & PS & THF & 20 & $\geq 30$ & Pores on the surface \\
\hline [45] & PLA & DCM & RT & 75 & $\begin{array}{l}\text { Pores on surface } \\
\text { diam }>5 \mu \mathrm{m}\end{array}$ \\
\hline [41] & PCL & Chloroform/DMF 80/20 & 21 & $\geq 50$ & $\begin{array}{l}\text { Pores on the surface } \\
\qquad(\text { diam }>5 \mu \mathrm{m})\end{array}$ \\
\hline [65] & PCL & $\begin{array}{l}\mathrm{CHCl3} \\
\text { or DCM }\end{array}$ & RT & $\geq 35$ & $\begin{array}{l}\text { Pore on surface } \\
\qquad \text { diam }=7 \mu \mathrm{m}\end{array}$ \\
\hline$[66]$ & PS & THF & 24 & $\geq 30$ & Pores on the surface \\
\hline [67] & PMMA & DMF & RT & $\geq 35$ & $\begin{array}{l}\text { Pores on surface } \\
\text { Diam }=2-5 \mu \mathrm{m}\end{array}$ \\
\hline [67] & PMMA & toluene & RT & $\geq 40$ & $\begin{array}{l}\text { Pores on surface } \\
\text { Diam }=1-8 \mu \mathrm{m}\end{array}$ \\
\hline
\end{tabular}




\begin{tabular}{|c|c|c|c|c|c|}
\hline [67] & PVC & DMF & RT & $\geq 60$ & $\begin{array}{l}\text { Pores on surface } \\
\text { Diam } \approx 0.6 \mu \mathrm{m}\end{array}$ \\
\hline [68] & PS & DCM/Ethanol 90/10 & 19 & $\geq 40$ & $\begin{array}{l}\text { Pores on the surface } \\
\text { diam }=5-6 \mu \mathrm{m}\end{array}$ \\
\hline [38] & PSU & $\mathrm{DMF}$ & $21-22$ & $\geq 40$ & $\begin{array}{l}\text { Pores on the surface } \\
\text { diam }=3-4 \mu \mathrm{m}\end{array}$ \\
\hline [9] & PS & THF & 40 & $\geq 60$ & $\begin{array}{l}\text { Pores on the surface } \\
\qquad(\text { diam }>10 \mu \mathrm{m})\end{array}$ \\
\hline [44] & PEI & NMP & 40 & $\geq 60$ & Pores on the surface \\
\hline [9] & PS & DMF & 20 & $\geq 30$ & $\begin{array}{l}\text { Internal porosity } \\
(\text { diam }=2-4 \mu \mathrm{m})\end{array}$ \\
\hline [47] & PS & THF & 20 & 22 & Internal porosity \\
\hline [69] & PS & THF/DMF & n.g. & 60 & $\begin{array}{c}\text { Internal porosity } \\
(\text { diam }=0.8 \text { to } 3 \mu \mathrm{m})\end{array}$ \\
\hline$[64]$ & PS & THF/DMF $1 / 4$ & 24 & 45 & Internal porosity \\
\hline [70] & PS & THF & 25 & $\geq 30$ & Internal porosity \\
\hline [71] & SAN & Ethanol/DMF 2/3 & 23 & 60 & Internal porosity \\
\hline$[72]$ & PI & DMF & RT & $\geq 30$ & Internal porosity \\
\hline$[44]$ & PEI & DMF or DMAc & 40 & $\geq 40$ & Internal porosity \\
\hline [73] & PCL & THF/DMF & RT & 25 & Branched fibers \\
\hline [74] & PVDF & $\mathrm{DMF} / \mathrm{THF}$ & 20 & 10 & Branched fibers \\
\hline [75] & $\begin{array}{c}\text { Anion } \\
\text { conducting } \\
\text { polymer }\end{array}$ & $\mathrm{DMF}$ & 24 & $\leq 30$ & Branched fibers \\
\hline
\end{tabular}




\subsection{Fiber shape and diameter}

The first effect of humidity that acts on the final fiber diameter is the discharge of the electrospinning jet at high humidity due to the increased amount of water molecules in the air resulting in the decrease of the amount of charges on the electrospun jet due to molecular polarization. The jet is consequently less vigorously subjected to whipping instabilities and less elongated. ${ }^{[76,67,77]}$ Secondly, humidity affects the solidification rate in different ways depending on the polymer-solvent systems, thus influencing the final diameter. On one hand, for a system composed of a hydrophobic polymer, the absorption of water in the jet leads to early solidification due to precipitation which results in thicker diameters and the suppression of bead-on-string morphologies that could be observed at high humidity (Figure 3a). ${ }^{[39]}$ On the other hand, for a hydrosoluble polymer or a polymer which can be plasticized by water, water absorption in the jet leads to delayed solidification either due to slower evaporation or to plasticizing of the polymer. In this case, the jet thins until it is subjected to the development of a capillary instability leading to the formation of beaded fibers (Figure 3b). ${ }^{[32]}$ 

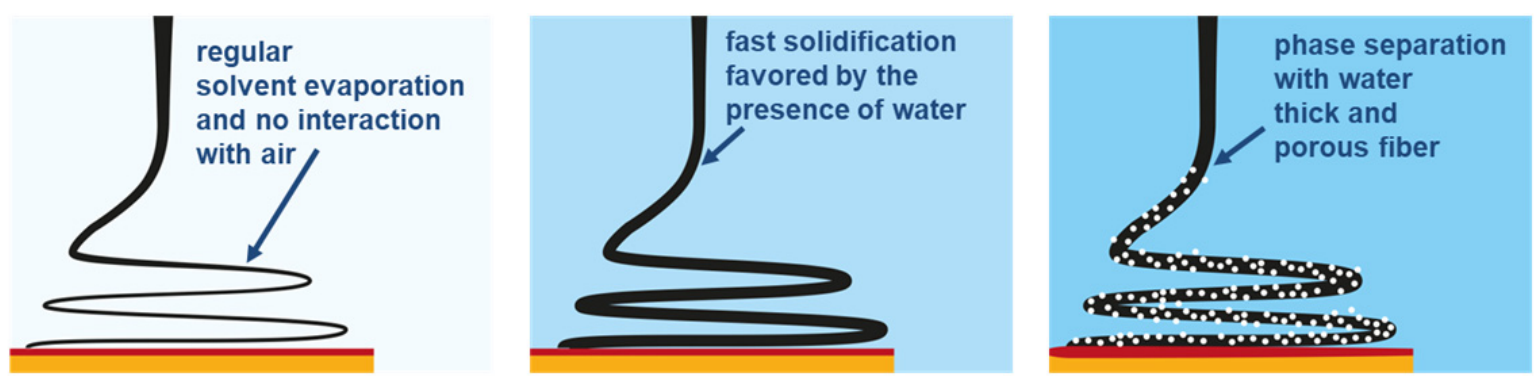

a) Increase of RH in the case of hydrophobic polymers
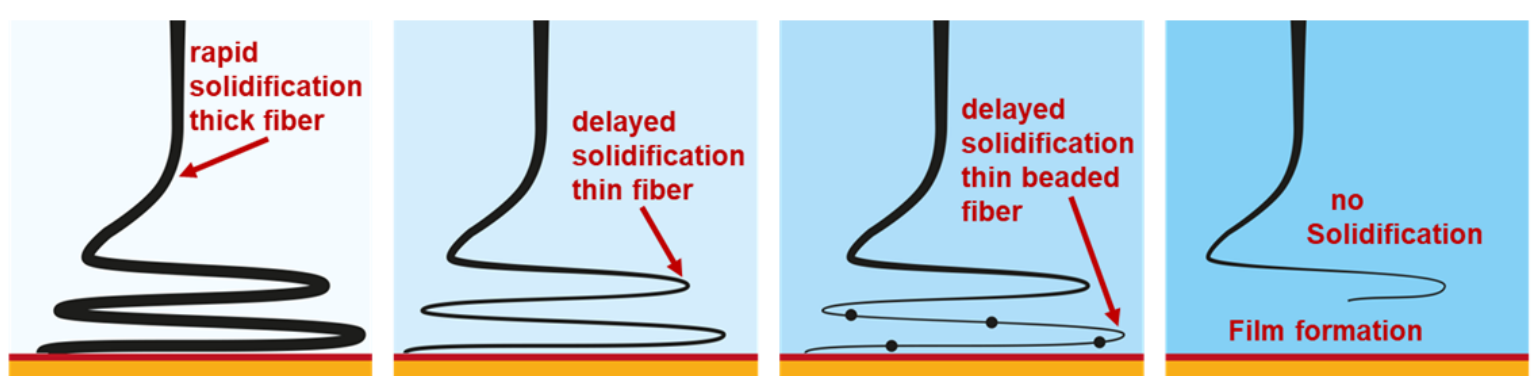

b) Increase of RH in the case of hydrosoluble polymers

Figure 3: Schematic representation of the effect of RH on the fiber diameter. a) Case of hydrophobic polymers. b) Case of hydrosoluble polymers in aqueous solvent systems and hygroscopic polymers which can be plasticized by water molecules at high RH.

Table 2 gives the evolution of the fiber diameters when increasing the relative humidity for various polymer/solvent systems given in the literature. For hydrophobic polymers, absorbed water molecules act as a non-solvent and fibers become thicker at high humidity. ${ }^{[38-40,54,71,78]}$ As an example, Kim et al. ${ }^{[39]}$ electrospun PS in THF/DMF and noted that the mean fiber diameter increased by $300 \%$ between $10 \%$ and $70 \% \mathrm{RH}$ at $20^{\circ} \mathrm{C}$. The increase of the fiber diameter has been reported for hydrophilic solvents such as DMF as well as hydrophobic ones such as chloroform in the case of PLA electrospinning. ${ }^{[35,78]}$ Pai et al. ${ }^{[9]}$ and Fashandi et al. $^{[9]}$ have shown that increasing the humidity could advantageously suppress the bead-on-string structure of PS fibers occuring when 
electrospinning was carried out below $15 \%$ of $\mathrm{RH}$. Indeed, at higher humidity, the solidification being accelerated, the development of the capillary instability is avoided.

On the contrary, when a hydrophilic polymer can be dissolved in water or in a hydrophilic solvent, ${ }^{[33,80]}$ the fiber diameter is thinner at high humidity. For instance, Pelipenko et al. ${ }^{[32]}$ have shown that the diameter of PVA fibers was decreased by four when increasing the relative humidity from $4 \%$ to $60 \%$ at $24^{\circ} \mathrm{C}$ due to slower evaporation of the water. By extension, bead-on-string fibers were obtained at high humidity in pure water solutions of $\mathrm{PVA}^{[32]} \mathrm{PEO}^{[33]}$ as well as more complex polymer systems such as $\mathrm{PEDOT} / \mathrm{PSS} / \mathrm{PEO}^{[81]}$ and for collagen fibers in phosphate-buffered saline/ethanol solvent system. ${ }^{[82]}$ The case of electrospinning of PEO in water solution was experimentally studied by Tripatanasuwan et al. ${ }^{[33]}$ as summarized in Figure $4 \mathrm{a}-\mathrm{b}$ showing that bead-on-string morphology appeared for $\mathrm{RH}$ greater than $52 \%$ and with a density of beads increasing with $\mathrm{RH}$. Cai and Gevelber ${ }^{[29,31]}$ studied in detail the behavior of PEO electrospun in water. They showed that the fiber average diameter is correlated with $\mathrm{RH}$ and the jet diameter $d_{j e t}$ in the straight jet domain which is directly linked with the electric and viscous force balance (see Figure $4 \mathrm{c}$ ). The relative humidity mainly impacts the process in the bending/whipping region where evaporation and jet stretching play a predominant role. The authors were also able to model the process in the bending region taking into account the mass and force balances. ${ }^{[31]}$ The prediction of the PEO fiber diameter as a function of the processing parameters and, among them RH, was possible with an accuracy of $6 \%$. Furthermore, the model demonstrates the conjugated effect of $\mathrm{RH}$ on both water evaporation and jet stretching. 

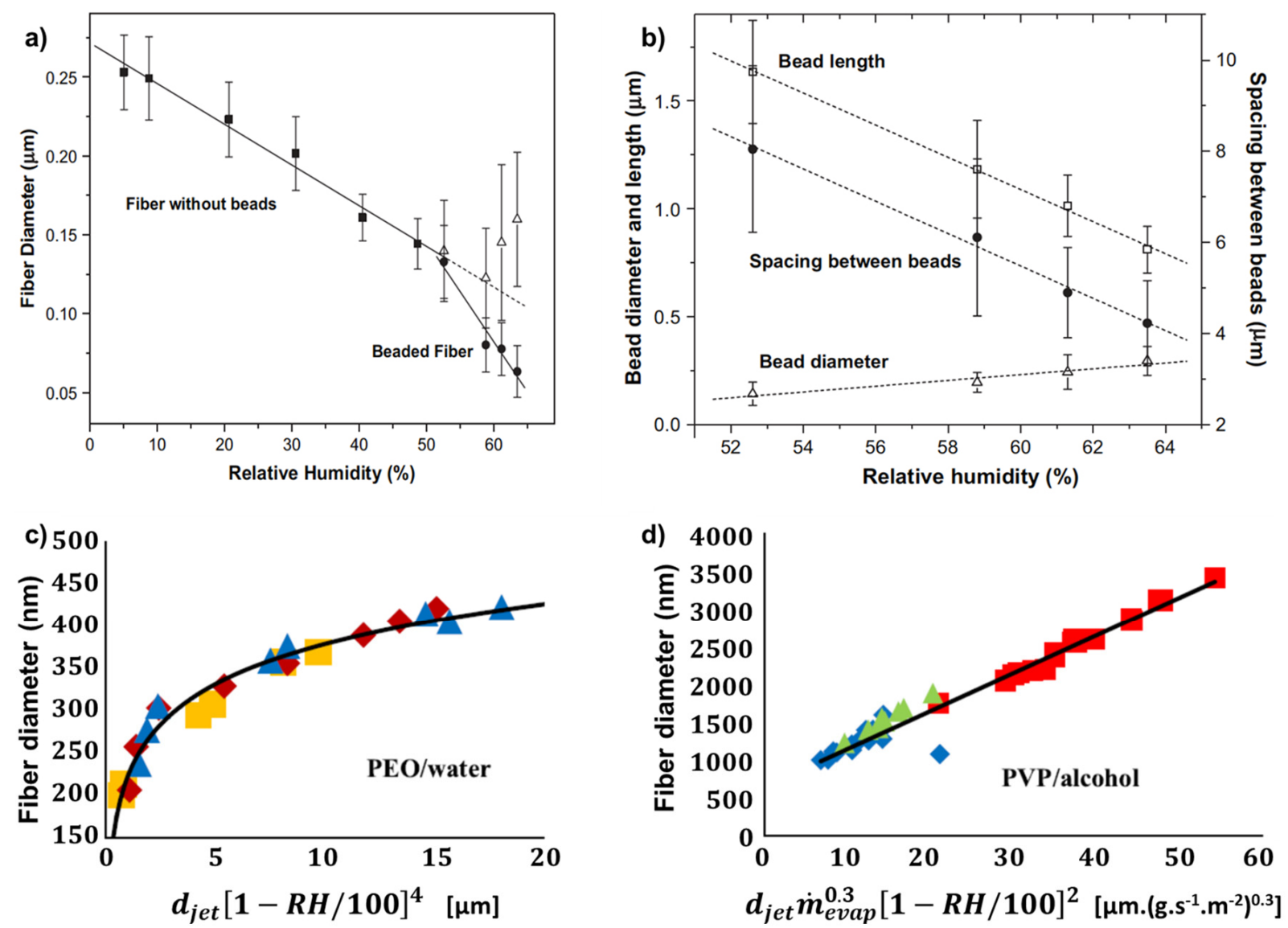

Figure 4: PEO nanofibers electrospun from water solutions at different relative humidity.

a) Average diameter of the fibers. Black squares: average diameter of regular fibers; black discus: fiber diameter between beads; open triangles: average fiber diameters calculated from the volume of the beads and their segments. b) Average bead diameters, bead length and distances between beads. a) and b) Reproduced with permission. ${ }^{[33]}$ Copyright 2007, Elsevier. c) Correlation of electrospun PEO fiber diameter with RH and jet diameter $\boldsymbol{d}_{\text {jet }}$ in the straight jet domain $3 \mathrm{~mm}$ beyond the apex of the Taylor cone. Water was used as solvent. d) Correlation of electrospun PVP fiber diameter with RH, $\boldsymbol{d}_{\boldsymbol{j} \text { et }}$, evaporation rate $\dot{\boldsymbol{m}}_{\text {evap }}$ per surface area for several non-aqueous solvents: blue diamonds (methanol), green tringle (ethanol) and red squares (butanol). c) and d) Reproduced with permission. ${ }^{\text {[31] }}$

Copyright 2016, Springer Nature. 
Cai and Gevelber ${ }^{[29,31]}$ studied also the electrospinning of PVP solubilized in various kinds of alcohols. Although, the evaporation of alcohol doesn't depend on RH, they observed a decrease of the fiber diameter with an increase of RH. A correlation of the fiber diameter with $\mathrm{RH}, d_{j e t}$ and $\dot{m}_{\text {evap,exp }}$ was obtained (see Figure $4 \mathrm{~d}$ ). Here, the evaporation rate $\dot{m}_{\text {evap,exp }}$, which in this case doesn't depend on $\mathrm{RH}$, was determined experimentally by weighting during time a solvent of contact area $A$ with air in an enclosed chamber. Although $\dot{m}_{\text {evap,exp }}$ was evaluated in a static mode (i.e. through evaporation by diffusion and not by convection as it happens during electrospinning), it was found that such solvent property has a significant influence on the evaporation and the net stretching force in the bending region. For the studied solvents, the authors have shown that RH has a greater impact on the process than the evaporation rate. In PVP/alcohol solution, an increase of RH may induce the absorption of water, a solvent of PVP, favoring the stretching of the jet in the bending region by a plasticizing effect. Similar results were confirmed in the case of PVP-based systems in alcohol/water solvent systems. ${ }^{[83]}$ Moreover, it was observed in the case of PVP-ethanol ${ }^{[48]}$ that the amount of water absorbed at high humidity can even be responsible of incomplete drying of the jet during the time of flight resulting in the collection of fused fibers and even a continuous film. Such effect could be avoided by increasing the emitter-to-collector distance. Hygroscopic polymers, such as polyamides (PA6, ${ }^{[52,53]}$ PA4.6 and PA6.9 ${ }^{[53]}$ ) in acetic acid/formic acid follow the same tendency. Indeed, as mentioned by the authors, the acid evaporation cannot be influenced by the amount of water in air and moreover, the low amount of absorbed water, even at high $\mathrm{RH}$, cannot be responsible of the acetic acid/formic acid phase diagram. ${ }^{[84]}$ Thus, the only explanation comes from the fact that the amount of absorbed water occurring at high $\mathrm{RH}$ is enough to drop the polyamide glass transition temperature. ${ }^{[85]}$ Such plasticizing 
effect allows the jet to be stretched for a longer time before reaching the collector, resulting in thinner fibers. The decrease of the fiber diameter with the increase of humidity was also observed for the needleless electrospinning of PA6 in an acetic acid/formic acid solvent system. ${ }^{[86]}$ However, in this case, it was also observed that no jet emission was achieved at RH lower than $40 \%$ due to air breakdown between the cylindrical emitter and the collector resulting from the combination of the low humidity and the very high voltage needed in needleless electrospinning. 
Table 2. Evolution of the fiber diameter of various polymer/solvent electrospinning systems as a function of RH. The diameter variation $=100 \times\left[\Phi_{\text {low RH }}-\Phi_{\text {high RH }}\right] / \Phi_{\text {low RH }}$

\begin{tabular}{|c|c|c|c|c|c|}
\hline Ref & Polymer & Solvent & $\begin{array}{c}\text { Temperature } \\
\left({ }^{\circ} \mathrm{C}\right)\end{array}$ & $\begin{array}{l}\text { Studied RH } \\
\text { range (\%) }\end{array}$ & $\begin{array}{c}\text { Diameter variation } \\
(\%)\end{array}$ \\
\hline$[32]$ & PVA & Water & 24 & $4-60$ & -76 \\
\hline 33] & PEO & Water & $21-22$ & $5-49$ & -43 \\
\hline [32] & PEO & $\begin{array}{c}3 \% \text { acetic acid } \\
\text { in water }\end{array}$ & 24 & $4-40$ & -48 \\
\hline [29] & PVP & ethanol & $21-24$ & $26-57$ & -50 \\
\hline [53] & PA4.6 & $\begin{array}{l}\text { Acetic acid/ } \\
\text { Formic acid }\end{array}$ & 21 & $10-70$ & -64 \\
\hline [51] & PA6 & $\begin{array}{l}\text { Acetic acid/ } \\
\text { Formic acid }\end{array}$ & 20 & $15-63$ & -57 \\
\hline [53] & PA6.9 & $\begin{array}{l}\text { Acetic acid/ } \\
\text { Formic acid }\end{array}$ & 21 & $10-70$ & -21 \\
\hline [80] & HA & DMF/Water & 25 & $8-18$ & -14 \\
\hline [71] & SAN & THF/DMF & 23 & $30-45$ & +6 \\
\hline [40] & CA & $\begin{array}{c}\text { Acetone/ } \\
\text { DMF/Ethanol }\end{array}$ & 25 & $20-70$ & +17 \\
\hline [87] & PAN/PEI & DMF & RT & $20-60$ & +56 \\
\hline [88] & PMMA & DMF & RT & $22-82$ & +88 \\
\hline [54] & PEI & NMP & 25 & $30-70$ & +124 \\
\hline [78] & PLA & Chloroform & 25 & $30-80$ & +129 \\
\hline [35] & PLA & DMF/DCM & 21 & $28-58$ & +169 \\
\hline
\end{tabular}




\begin{tabular}{|c|c|c|c|c|c|}
\hline [39] & PS & THF/DMF 6/4 & 20 & $10-70$ & +192 \\
\hline [38] & PSU & DMF & 22 & $0-60$ & +211 \\
\hline [89] & PCL & $\begin{array}{l}\text { Acetic acid/ } \\
\text { Formic acid }\end{array}$ & 25 & $35-65$ & +237 \\
\hline [38] & PAN & DMF & 22 & $0-60$ & +320 \\
\hline
\end{tabular}

\subsection{Wrinkled, rough and porous fibers}

For hydrophobic polymers in various solvents, depending on humidity, electrospinning can lead to wrinkled or rough fibers, ${ }^{[79]}$ as well as fibers having pores in their core and/or in their surface ${ }^{[9,45,46,90-92]}$ (Table 2). For example, Li et al ${ }^{[93]}$ have recently studied the case of PLA/chitosan electrospun in DCM/DMAc. They showed that the coverage of pores on the fiber surface as well the pore size increased significantly with humidity. Moreover, porous fibers are generally observed for thick fibers having diameter in the range of few microns as shown in the section devoted to porous structure in Table 1. Figure 5 shows different kind of porous fibers. The surface and internal morphology of the fibers is formed at the moment of the fiber solidification, which is due either by VIPS, TIPS, breath figure self-assembly or non-solvent induced phase separation (NIPS) or a combination of these mechanisms. 

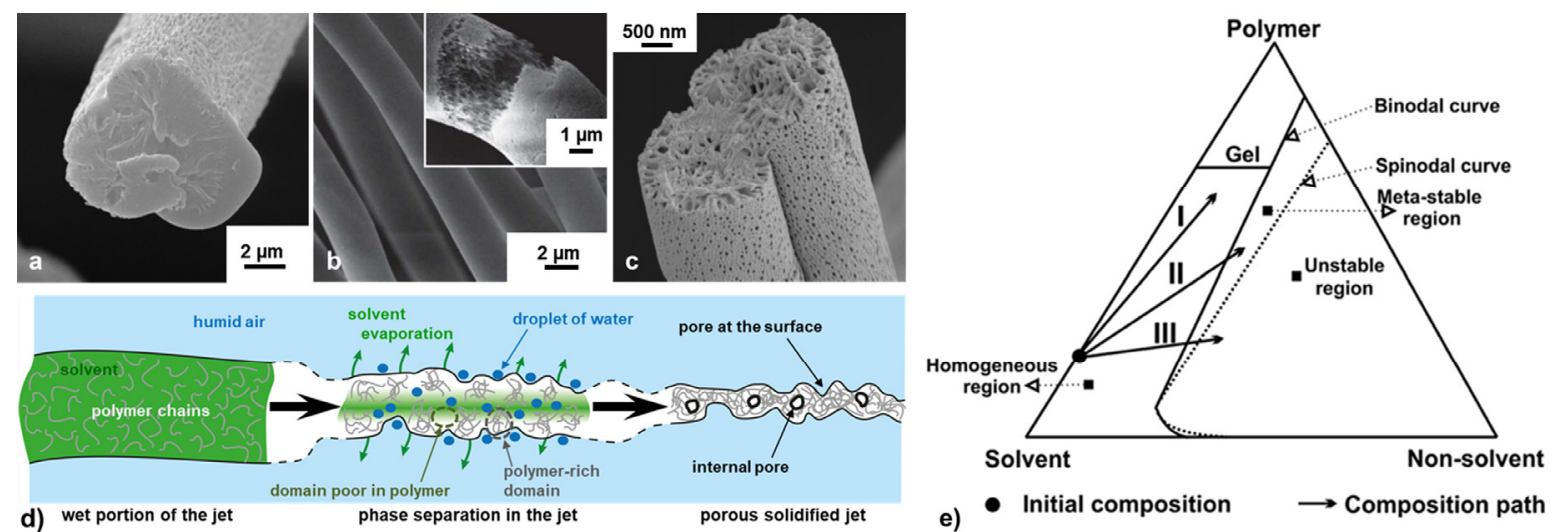

Figure 5. a) PS fibers composed of a porous surface and a solid inside electrospun in THF at $40^{\circ} \mathrm{C}$ and $60 \% \mathrm{RH}$. Reproduced with permission. ${ }^{[9]}$ Copyright 2012, Elsevier. b) PS fibers composed of a smooth surface and internal pores electrospun in THF and DMF at $24^{\circ} \mathrm{C}$ and 45\% RH. Reproduced with permission. ${ }^{[64]}$ Copyright 2009, Royal Siciety of Chemistry. c) Fibers containing PS, sorbitan monooleate and fluorescein sodium salt composed of a porous surface and internal pores electrospun in DMF and chloroform at $20^{\circ} \mathrm{C}$ and $60 \% \mathrm{RH}$. Reproduced with permission. ${ }^{[42]}$ Copyright 2015, Elsevier. d) Pore formation along the electrospun jet in the case of a hydrophobic polymer at high RH. e) Schematic representation of polymer-solvent-non solvent phase diagram and the possible composition paths that can undergo fibers during electrospinning. Reproduced with permission $\cdot{ }^{[9]}$ Copyright 2012, Elsevier.

Pore formation by VIPS. VIPS is related with the diffusion of vapor inside the fibers, which is a non-solvent for hydrophobic polymers. At high humidity and for solvents that are miscible with water, such as THF or DMF, the mass transfer of water inside the fiber will lead to a change of composition of the polymer solution that can be described by composition paths on the solvent/non-solvent/polymer phase diagram (Figure 5e). If the solvent evaporation rate is faster than water absorption, the composition path I is followed and no phase separation occurs in the solution. Fashandi et al. ${ }^{[9]}$ have shown that this was 
the case of PS electrospun from THF solutions because THF evaporation is rapid but also because the homogeneous region was larger for PS-THF-water solutions with up to 5\% water. Therefore for PS electrospun from THF solution, the core of the fibers is compact. ${ }^{[9,39,47]}$ If the composition path followed during electrospinning crosses the binodal curve to end in the metastable region (path II), phase separation occurs via nucleation and growth mechanism, leading to a porous core with isolated cellular pores. Finally, if the composition path crosses the spinodal curve and ends up in the unstable region, phase separation occurs via spinodal decomposition, leading to co-continuous interconnected networks of pore in the core of the fibers. This has been observed for PS solutions in DMF. ${ }^{[43,47,79,64]}$ In this case, evaporation of DMF is slower and the homogeneous region of the phase diagram is small, precipitation occurs with less than $0.2 \%$ of water in a PS/DMF solution. Lu et al. ${ }^{[47]}$ observed fibrils inside the core of PS fibers electrospun from DMF. This is probably due to the bicontinuous phase separation undergone during electrospinning, combined with the elongation of the structure. Porous fibrous core have also been observed for poly(imide) in $\mathrm{DMF},{ }^{[72]}$ poly(ether imide) in DMF or DMAc, ${ }^{[4]}$ or styrene/acrylonitrile copolymers in ethanol/DMF $2 / 3 .{ }^{[71]}$

The rate of solvent evaporation depends on the vapor pressure of the solvent. Evaporation of the solvent combined to slow diffusion may lead to the formation of polymer concentration gradients with a high concentration near the surface, leading to thin solid skin around the jet. This skin is then subjected to compressive radial stresses that cause buckling instabilities, leading to wrinkled or rough fibers. Pai et al. ${ }^{[79]}$ have linked the appearance of rough fibers with the drying time and the buckling time first described by Pauchard et al. ${ }^{[94]}$ for polymer solution droplets. The drying time $\left(t_{D}\right)$ is the time needed for the fiber to dry completely. It is proportional to the diameter of the fibers inversely 
proportional to the evaporation rate. The buckling time $\left(t_{B}\right)$ depends on the mutual diffusion coefficient of the polymer-solvent system, the solution concentration and is inversely proportional to the square of evaporation rate. Smooth fibers are obtained when $t_{B}>t_{D}$, whereas rough fibers are formed if $t_{B}$ is comparable to $t_{D}$. For example, in the case of PS solution in THF, the appearance of wrinkled fibers depends on the diameter of the fibers, or the molecular weight of the polymer. Thicker fibers tend to collapse because the time needed for complete drying of the fiber is larger while fibers made from higher molecular weight collapse because, the diffusion coefficient of the polymer-solvent system is low, which result in lower $t_{B}$. In the case of PS fibers electrospun from DMF solutions, wrinkled fibers were also observed at low relative humidity. However, when $\mathrm{RH}$ is high enough, phase separation inside the fibers is faster than both buckling and drying due to absorption of water, leading to smooth cylindrical fibers with the presence of pores within the interior of the fibers. ${ }^{[9,79]}$

Pore formation through breath figure self-assembly. Rapid evaporation of the solvent can also lead to an important temperature decrease due to the latent heat loss, leading to the condensation of water vapor at the surface of the fibers. After complete evaporation, pores at the surface of the fibers left by the water droplets, and also called breath figure self-assembly, ${ }^{[95]}$ can be observed. Yazgan et al. ${ }^{[65]}$ linked the transition from smooth to rough fibers with the dew point $\left(T_{d p}\right)$ and the wet-bulb temperature $\left(T_{w b}\right)$, where the wetbulb temperature refers to the minimum temperature that the polymer solution reaches during evaporation due to latent heat loss. Water condensation on the fiber occurs when $\mathrm{T}_{\mathrm{dp}}-\mathrm{T}_{\mathrm{wb}}>0$. They observed this phenomenon for PCL fibers electrospun from dichloromethane or chloroform (Figure 6). Similar results were obtained for PCL electrospun in other various solvent systems ${ }^{[96]}$ and for PS, PMMA, PLA and PVDF fibers 
electrospun from fast evaporating solvent such as THF, acetone, dichloromethane or chloroform. ${ }^{[39,45,46,66,68,97]}$ In the case of PVDF electrospun in acetone, Zaarour et al. ${ }^{[97]}$ observed that not only the number of pores increases with RH but also their size (Figure 6C-F). The evaporation of acetone, a highly volatile solvent, cools the surface of the jet which leads to the condensation of water into droplets. The evaporation of these water droplets being more difficult at high $\mathrm{RH}$, their coalescence in the form of macro-droplets by nucleation growth is favored and results in the formation of macro pores on the surface of the fibers (Figure 6F). Casper et al. ${ }^{[66]}$ showed that the size of the pores created on the surface of PS fibers, electrospun from THF, increased with the relative humidity, but surprisingly, also with the molecular weight of the polymer. It was concluded that in this case, both the condensation of water and phase separation on the surface of the fibers, due to the decrease of temperature probably occurs at the same time.

Pore formation by TIPS. Solvent evaporation during electrospinning leads to a decrease of the temperature of the jet. Thus, if the jet temperature is enough lower, the polymer may reach the limit of solubility inducing its precipitation and the formation of pores or wrinkle surface. TIPS may happen at low RH as observed in the case of wrinkled PMMA fibers electrospun at $12 \% \mathrm{RH}^{[98]}$ and for PVDF fibers electrospun at $25 \% \mathrm{RH} .{ }^{[99]}$ Because TIPS is not affected by humidity, it is not studied in the present review.

Pore formation by NIPS. Finally, complex porous morphologies (Figure 6a-b) of the fibers can also be obtained by using a mixture of two different solvents with different boiling temperature, or affinity with the polymer, as suggested by Yazgan et al. ${ }^{[65]}$ In the present review we focus on water as the non-solvent inducing the phase separation which creates the pores on hydrophobic polymer fibers. However, it is worth noting that porous fibers can also be obtained from non-solvent-induced phase-separation of ternary systems 
(NIPS $)^{[100]}$ by the incorporation of a non-solvent in the electrospinning solution, but this is not the subject of the present review.

a
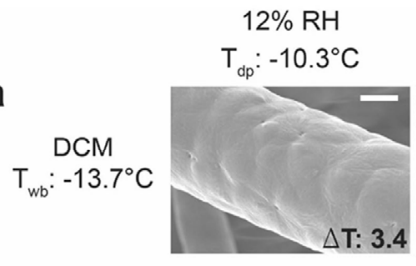

b

$\underset{\mathrm{T}_{\mathrm{wh}}:-4.6^{\circ} \mathrm{C}}{\mathrm{CHCl}_{3}}$
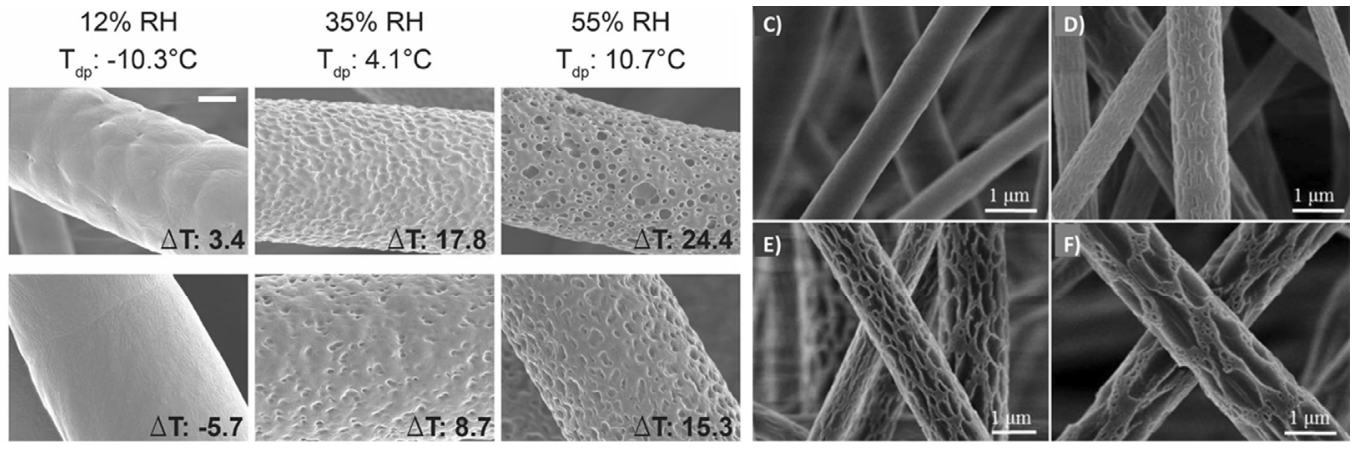

Figure 6. a) and b ) Morphologies of electrospun PCL fibers spun with DCM and chloroform $\mathrm{CHCl}_{3}$ : SEM micrographs and corresponding $\Delta \mathrm{T}=\mathrm{T}_{\mathrm{dp}}-\mathrm{T}_{\mathrm{wb}}$ of a) DCM (upper

row) and b) $\mathrm{CHCl}_{3}$ (lower row) for PCL fibers electrospun at $12 \% \mathrm{RH}, 35 \% \mathrm{RH}$ and $55 \%$ RH. Scale bar: $2 \mu \mathrm{m}$. a) and b) Reproduced under the terms of the Creative Commons CC BY license. ${ }^{[65]}$ Copyright 2017. C-F) Morphologies of PVDF electrospun in acetone under various RH. C) to F) Reproduced under the terms of the Creative Commons CC BY license. ${ }^{[97]}$ Copyright 2018.

\subsection{Branched fibers}

Observed for the first time by Reneker et al. ${ }^{[101]}$ in the case of PEO, branching of electrospun PCL fibers was then studied by Yarin et al. ${ }^{[73]}$ Branched fibers result from the ejection of secondary jets in radial direction from the surface of the primary electrospun jet. Based on an electrohydrodynamic model taking into account the effect of the electric Maxwell stresses and the surface tension on the deformation of the electrospun jet, it was shown that undulations develop periodically along the surface of the primary jet. When the electric field is high enough, instabilities occur in the vicinity of areas of highest local surface curvature from which the secondary jets emanate forming thus lateral branches 
along the fibers. ${ }^{[73]}$ The model was qualitatively compared to the electrospinning of PCL at various voltages. It is worth noting that the relative humidity was fixed at a low value of $25 \%$ but its effect was not discussed by the authors. However, as the branching effect is directly related to the amount of charges carried by the jet, it may be triggered from the control of humidity during electrospinning. Thus, very recently, Zaarour et al. ${ }^{[74]}$ observed that when a small amount of cationic surfactant (tetrabutylammonium chloride) is added in a formulation of PVDF solubilized in a DMF/THF solvent system, fiber branching can occur if electrospinning is carried out under low $\mathrm{RH}=10 \%$. These processing conditions (i.e. low RH and addition of cations) were thus ideal to insure a high level of charges at the surface of the primary jet which is necessary for the emanation of secondary jets resulting finally to the fabrication of branched PVDF fibers. Branching effect was also observed by Halabi et al. ${ }^{[75]}$ in the case of a ionomer, a quaternary ammonium functionalized aromatic poly (p-phenylene oxide))-based polymer, electrospun in DMF. In this case, branching occurred at $\mathrm{RH} \leq 30 \%$ with much more branches at $30 \%$ than at $20 \%$. No branching was observed when electrospinning was conducted at $\mathrm{RH} \geq 40 \%$. Once more, the formation of branches is due to the emanation of secondary jets which is favored by a high level of free charges at the surface of the jet. Such condition was probably fulfilled thanks to the presence of the ionomer in the solution and the low $\mathrm{RH}$ which avoids the rapid discharging of the jet. The fact that less branches were observed when the RH was set at $20 \%$ was explained by the rapid solidification of the surface of the primary jet avoiding thus the efficient emanation of secondary jets. 


\subsection{Effect of humidity on the fiber crystalline structure}

Very recently, researchers started to pay attention on the effect of humidity during electrospinning on the resulting crystalline structure of the processed material. The case of PVDF which can crystalize in four phases namely $\alpha, \beta, \gamma$ and $\delta$, has been studied from this point of view. In particular, the all-trans $\beta$-phase was investigated with special care as the opposite arrangement of fluorine and hydrogen atoms exhibits the largest dipole moment resulting in piezoelectric and triboelectric properties. ${ }^{[102]}$ It was observed that an increase of RH enhances significantly the amount of $\beta$-phase when PVDF is electrospun in various solvent systems such as DMF/THF, ${ }^{[99]}$ acetone/DMF ${ }^{[103,104]}$ and DMAc/acetone. ${ }^{[105]}$ Kong et al. propose that the increase of the $\beta$-phase amount with $\mathrm{RH}$ may be due to delayed solidification of the core of the electrospun jet. ${ }^{[104]}$ Because electrospinning of PVDF was carried out in acetone/DMF, it allows the fast evaporation of acetone leading to the decrease of the temperature of the jet surface promoting water condensation and thus the PVDF precipitation/solidification at the jet surface. The as-formed solid skin delays the evaporation of DMF contained in the jet core retarding thus the whole solidification process as compared when electrospinning is carried out at low RH. The authors argued, by analogy with solution-cast PVDF films, ${ }^{[106]}$ that delayed solidification under high RH may provide more time for PVDF $\beta$-phase to nucleate and grow. Thus, PVDF fibers electrospun in an environment having a high RH should contain a larger ratio of $\beta$-phase than those elaborated under dry condition. However, the processing characteristic time is much shorter for electrospinning than for solution casting for which the evaporation rate, dominated by the solvent diffusion coefficient, is low enough to affect the crystallization kinetic of $\mathrm{PVDF}^{[106]}$. Szewczyk et al. ${ }^{[105]}$ mentioned that the increase of the $\beta$-phase amount with RH corroborates with the wrinkling and the formation of pores in the PVDF 
fibers. Knowing that the $\beta$-phase content is enhanced by stress, ${ }^{[107]}$ they hypothesized that the stresses, induced at high RH by the local deformations occurring at the vicinity of the wrinkles and pores, may favor the crystallization of $\beta$-phase. Finally, such piezoelectric PVDF electrospun fibers having an optimized $\beta$-phase content exhibited high power density interesting for energy harvesting applications. ${ }^{[104,105]}$

It was also observed that humidity affects the crystallization of cyclodextrin electrospun fibers. ${ }^{[108]}$ Cyclodextrins are cyclic oligosaccharides with 6,7 or 8 glucose units (respectively $\alpha-C D, \beta-C D$ and $\gamma-C D$ ) for which it has been demonstrated that electrospun fibrous scaffolds can be obtained without the help of any template polymer thanks to favorable supramolecular interactions occurring in the processed solution. ${ }^{[109,110]}$ Yoshida and Sakuragi ${ }^{[108]}$ evidenced by XRD that electrospinning of $\alpha-C D$ in HFIP leads to amorphous fibers for $\mathrm{RH}<60 \%$ whereas fibers with crystalline channel-type structures were obtained at higher RH. A degree of crystallinity of $76 \%$ was achieved when electrospinning was carried out at $\mathrm{RH}=80 \%$. This result is surprising as channel crystals are mostly observed when $\alpha-C D$ are electrospun in the form of necklace-like supramolecular assemblies such as polyrotaxane. ${ }^{[111,112]}$ As $\alpha-C D$ is highly water soluble, the increase of RH may delay and favor the strong stretching of the electrospun jet during its solidification leading to channel-type crystallization. ${ }^{[8]}$ Similarly, crystallization of $\beta$ CD was achieved at high RH but with a mixture of channel and cage-type structures as reported for the preparation $\beta-\mathrm{CD}$ films by solution-casting in HFIP. ${ }^{[113]}$ 


\section{Effect of humidity at the scale of the mat}

Variations in humidity can also have consequences on the structure at the scale of the electrospun nonwoven mats. With a variation of $\mathrm{RH}$, fibers can stick together or be differently oriented. Mats can turn into films or differently gain a cotton-like structure. Finally, nanonets may be found in the structure of mats between the fibers.

\subsection{Effect of humidity on the bending instabilities}

As previously discussed, a modification of $\mathrm{RH}$ affects the amount of charges carried at the jet surface responsible to the whipping movements and which in turn affects the way the jet is deposited on the collector. As an example, when an aqueous solution of gelatin is electrospun, an important decrease of the bending instabilities is observed by increasing RH. ${ }^{[14]}$ The same effect was characterized in the case of PVDF by measuring the average diameter of the deposited mat. ${ }^{[15]}$ The increase of RH results thus in a focused deposition of the nanofibers. However, such effect was negligible as observed by Cai and Gevelber in the case of electrospinning PEO in water. ${ }^{[29]}$ Indeed, the whipping movements are the result of the competition between several phenomena: i) the electric force due the surface charges, ii) the surface tension which may stabilize the jet and iii) the viscoelastic properties of the jet. ${ }^{[30]}$ The effect of RH on the whipping movements is thus complex and special care must be taken when the control of the deposit is of prime importance. Finally, the control of $\mathrm{RH}$ must be considered when the precise manipulation of the jet by controlling the buckling instabilities ${ }^{[116]}$ is necessary. 


\subsection{Fiber-sticking and film formation}

This phenomenon happens when the solvent is not completely evaporated leading to the deposition of fibers which are not completely solidified before reaching the collector. As previously discussed, the cases for which humidity postpones the solvent evaporation correspond to the cases when the solvent is water or when it is hydrophilic. Consequently, the small amount of residual solvent that remains on the fibers enables them to stick together. Fibers-sticking conveys electrospun mats better mechanical properties. ${ }^{[80,117]}$ For example, Ding et al. ${ }^{[117]}$ observed this behavior at 75\% RH with PAA fibers electrospun in a mixture of water and ethanol at $25{ }^{\circ} \mathrm{C}$. When the solidification of the fibers is even slower, they cannot solidify any more, fuse together and turn into a film..$^{[0,48,32,118-120]}$

\subsection{Fiber alignment and structuration}

\subsubsection{Fiber alignment and structuration using patterned collectors}

Generally, electrospinning leads to the elaboration of a nonwoven mat due to the pseudorandom deposition of the charged jet resulting from the electro-hydrodynamic bending instabilities. ${ }^{[101]}$ However, several strategies have been proposed to fabricate mats with aligned or structured fibers. For example, when patterned collectors made of protrusions are used, it is possible to induce a regular structuration of the deposited fibers. ${ }^{[121-124]}$ The structuration mechanism relies on the charges remaining on the portions of the fibers hanging in air between the protrusions. ${ }^{[124]}$ These suspended charged fiber strands induce repulsive forces acting on the incoming electrospun jet whereas the portions of fibers which are directly in contact with the protrusions of the collector are discharged inducing thus an attractive force. ${ }^{[125]}$ These repulsive and attractive areas, regularly arranged, 
according to the design of the patterned collector, form the so-called electrostatic template. ${ }^{[126]}$ Under certain conditions, the electrostatic template can be maintained for a long time during the electrospinning deposition allowing the fabrication of thick $3 \mathrm{D}$ structured constructs. ${ }^{[125,127]}$ However, if the charges of the suspended portions of fibers are rapidly released during the process, the electrostatic template is lost and no structuration occurs. This rapid charge release happens when the deposited fiber is enough conducting (e.g. when conducting polymers such as polyelectrolytes or polysaccharides are electrospun or if the deposited fiber contains a residual amount of a conducting solvent). As an example, Li et al. ${ }^{[128]}$ have shown that humidity is a major parameter affecting the fiber structuration. Indeed, they observed that low humidity $(\sim \mathrm{RH}=20 \%$ at room temperature) was necessary to insure PVP fiber alignment with patterned collectors. Higher RH induced a rapid discharge of the deposited fibers through the ions present in the air and the loss of the electrostatic template favoring the fiber structuration.

\subsubsection{Honeycomb-like structures through fiber self-assembling}

Structuration in the form of honeycomb-like structures can also occur through a selfassembling mechanism when the deposited fibers has a bimodal distribution of the diameter (due to the formation of bead-on-string structure or due to heterogeneities in the polymer solution). ${ }^{[129]}$ Indeed, when the fiber reaches the collector, the widest portions of the fiber touch the collector whereas the thinnest parts keep suspended in the air avoiding their charge release. Similarly to the structuration induced with patterned collectors, an electrostatic template is formed favoring the self-assembling of the incoming fiber and its preferential deposition on the fibrous domains in direct contact with the collector. Ye et 
al. $^{[130]}$ observed such honeycomb structures in the case of chlorinated polypropylene solubilized in a xylene/cyclohexanone solvent system when electrospinning is carried out at relatively low RH (Figure 7a-b). However, the honeycomb self-assembling process was lost for the highest studied $\mathrm{RH}=75 \%$ (Figure $7 \mathrm{c}$ ). Under such humid condition, the water molecules condensed at the jet surface may favor the charge release thanks to molecular polarization and charge transfer ${ }^{[38]}$ avoiding thus the formation of the electrostatic template, the sine qua non pre-requisite condition for the building of self-assembled structures. Similar results were obtained by Liang et $\mathrm{al}^{[118]}$ in the case of PEO electrospinning.
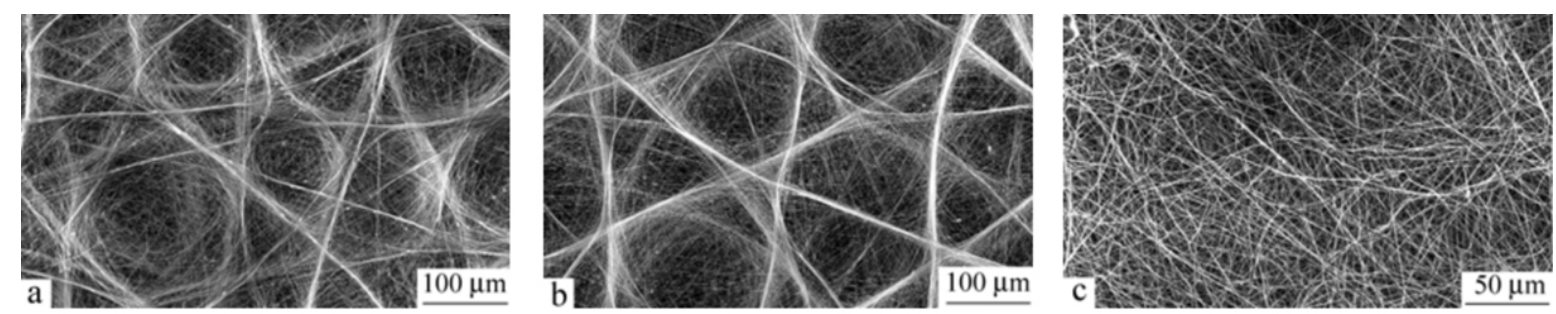

Figure 7. SEM of chlorinated polypropylene fibers. Effect of $\mathrm{RH}$ on the formation by self-assembling of honeycomb fibrous structures. a) $\mathrm{RH}=45 \%$, b) $\mathrm{RH}=55 \%$ and c) $\mathrm{RH}$ $=75 \%$. a) to c) Reproduced with permission. ${ }^{[130]}$ Copyright 2011, Springer Nature.

\subsubsection{Formation of yarns during the bending instabilities}

The group of Ding studied how humidity can induce fiber alignment during the bending instabilities. ${ }^{[131-133]}$ They applied this phenomenon in order to fabricate PMIA yarns with highly aligned fibers and robust mechanical strength. Whereas a random deposition was observed at low $\mathrm{RH}=35 \%$, highly aligned fibers were obtained at $\mathrm{RH}=55 \%$ (Figure $8 \mathrm{a}$ b). At high $\mathrm{RH}=65 \%$, a fluffy mesh with partially aligned fibers was obtained (Figure 8c). The authors proposed that the effect of RH on the formation of PMIA yarns can be 
due to charge loss and phase separation (Figure 8e). It was observed by optical photography (Figure $8 \mathrm{f}-\mathrm{i}$ ) that the whipping instabilities narrowed with increasing the $\mathrm{RH}$ from $35 \%$ to $55 \%$. Then, for the highest $\mathrm{RH}=65 \%$ a broadening of the whipping movement is observed. PMIA is highly hydrophilic (but not water soluble) as well as the components of the solvent system which is an $\mathrm{LiCl} / \mathrm{DMAc}$ ionic liquid. Consequently, the humidity impacts significantly the flying and solidification of the electrospun jet. When increasing $\mathrm{RH}$ from $35 \%$ to $55 \%$, the large amount of water molecules surrounding the electrospun jet favors the charge release thanks to molecular polarization and charge transfer. ${ }^{[38]}$ The electrostatic repulsion at the origin of the whipping movements are thus narrowed at high $\mathrm{RH}$ favoring the formation of yarns. Because water molecules act as a non-solvent of PMIA, the water droplets that may form at the jet surface for the highest studied RH should induce the rapid solidification of the jet decreasing the efficient formation of yarns while favoring the formation of a cotton-like structure with poor fiberfiber interactions. Similarly, PPV electrospun yarns were obtained by Okuzaki et al. ${ }^{[134]}$ 

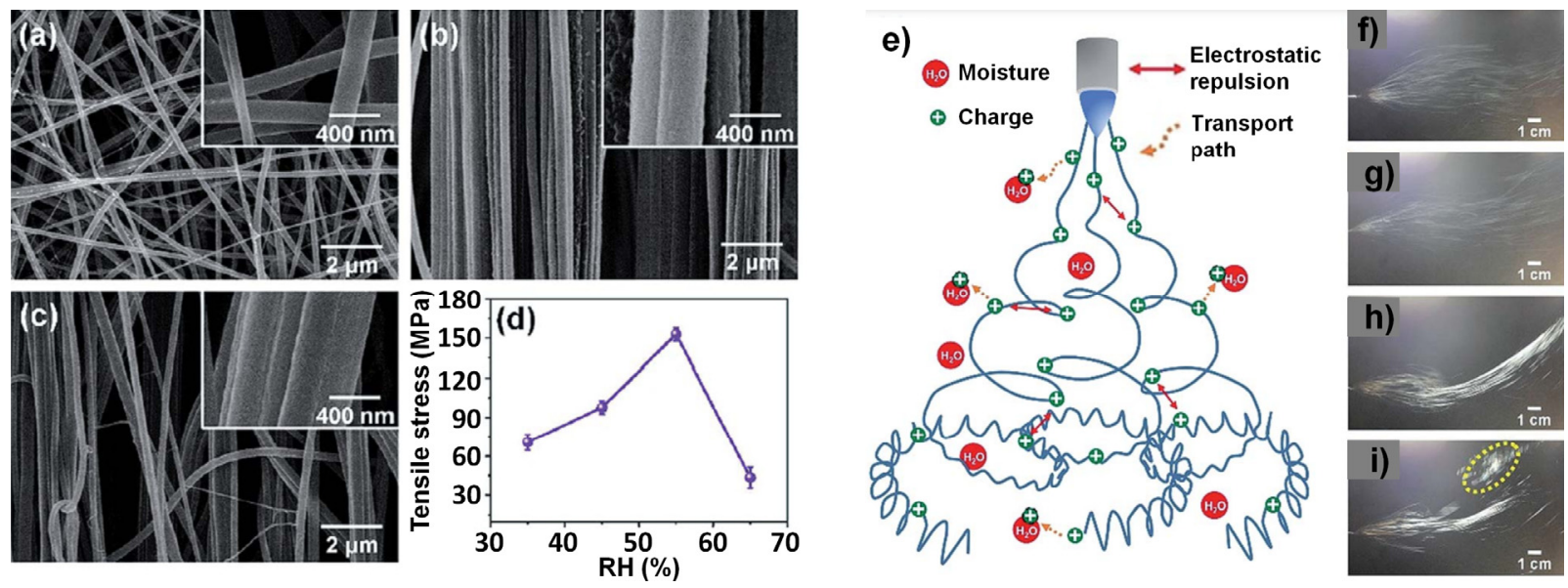

Figure 8. FE-SEM images of PMIA nanofibrous membranes fabricated from $15 \mathrm{wt} \%$ PMIA solution at RH of (a) 35\%, (b) 55\%, and (c) $65 \%$. (d) The tensile stress of relevant membranes. (e) Schematic diagram illustrating the possible formation mechanism of nanofibers with different alignment degree during electrospinning process. Optical images of charged jet behavior at different RH of (f) $35 \%$, (g) $45 \%$, (h) $55 \%$, and (i) $65 \%$. a) to i)

Reproduced with permission. ${ }^{[131]}$ Copyright 2011, Royal Society of Chemistry.

\subsection{Cotton-like 3D structures and mats with large pores}

Electrospinning is a process that generally allows only the fabrication of thin nanofibrous mats having a thickness lower than few hundreds of microns. However, it has been reported that under specific conditions cotton-like structures can be obtained with thickness as high as few $\mathrm{cm}$. Such 3D structures can be obtained only when the electrostatic charges cannot efficiently dissipate from the surface of the deposited fiber to the collector. Fluffy cotton-like structures of several $\mathrm{cm}$ height were obtained by Li and Long $^{[135]}$ for the electrospinning of PS and PVP/nitrate, by Su et al. ${ }^{[136]}$ for electrospinning of polyborosiloxane and by Bonino et al. ${ }^{[137]}$ and Jeong et al. ${ }^{[138]}$ in the case of alginatebased systems (Figure 9a-f). More recently, it has been shown that PVDF electrospun in DMF under very high humidity $(\mathrm{RH} \geq 90 \%$ ) can also result in cotton-like 3D 
structures. ${ }^{[103]}$ Huang et al. ${ }^{[38]}$ proposed a mechanism leading to the formation of such structures in the cases of PAN and PSU nonwovens also electrospun from DMF solutions. Fast solidification induces rare fiber-fiber bounding in nonwovens limiting thus the whole charge dissipation and finally yielding a cotton-like structure. More precisely, residual solvent is needed to allow fiber-fiber adhesion, and as because of fast solidification the amount of residual solvent on the surface of the fibers is reduced, very few fibers can bind together increasing thus fiber-fiber charge repulsions. Similar results were obtained in the case of PS fibers prepared from a DMF/THF solvent mixture. ${ }^{[139,140]}$

For aqueous polyelectrolyte solutions, as observed for alginate, ${ }^{[137]}$ the mechanism leading to the formation of the cotton-like structures is more complex because humidity plays an important role on the evaporation rate, on one hand, and on the amount of charges carried by the deposited fiber, on the other hand (Figure 9a-f). Indeed, an increase of RH allows to keep the charged groups ( $\mathrm{R}-\mathrm{COO}^{-}$and $\left.\mathrm{Na}^{+}\right)$of the polyelectrolyte in their dissociated form favoring thus the fiber-fiber repulsions necessary for the formation of the cotton-like structure. However, poor evaporation rate is experienced for the highest RH leading to beaded fibers as well as a better fiber-fiber contact leading to efficient charge release and finally avoiding the formation of the cotton-like structure.

\subsection{Nanonets}

Relative humidity can also influence the apparition of nanonets (also called nano-webs) (Figure 9g-i). This hierarchical fibrous structure corresponds in nets of very thin nanofibers with diameter in the range of few tens of nm suspended between larger fibers with diameter in the range of several hundreds of nm to few microns. Different theories could explain the formation of this structure and no consensus has been yet reached. ${ }^{[63]}$ 
Ding et al. ${ }^{[117,141]}$ hypothesized that nanonets are formed from charged microsized droplets generated during the process of electrospinning which are subjected to fast phase separation (Figure 9g). The presence of such droplets electrosprayed simultaneously during the deposition of the electrospun fiber was indeed already demonstrated and discussed by Rutledge et al. ${ }^{[142]}$ In fact, Zhang et al. ${ }^{[143]}$ suggested that above a critical charge-to-mass ratio microsized droplets are produced simultaneously with the electrospun jet. The so-formed microsized droplets are then deposited on the fibers in the form of suspended thin films which can split into nanonets by phase separation between polymer and solvent. The splitting into nanonets by phase separation can only happen when fast evaporation of the solvent occurs. Consequently, humidity plays an important role in the formation of nanonets as it affects the evaporation rate and especially for hydrophilic polymers. Indeed, in this case, because polymers such as PAA $^{[143]}$ or gelatin $^{[141]}$ are solubilized in aqueous solvents, fast phase separation and thus efficient nanonets formation can only happen at low RH. However, the authors observed that the addition of $\mathrm{NaCl}$ salt in the solution ensured the formation of nanonets at higher humidity but the mechanism behind this observation is not clear. In the case of hydrophobic polymers, as observed for PU, ${ }^{[144]}$ it was not observed an influence of humidity on the nanonet formation. However, for more complex systems such as electrospinning of PCL/wool keratin solubilized in formic acid, the presence of wool keratin as well as the decrease of RH were mandatory to induce the formation of nanonets. ${ }^{[145]}$ 

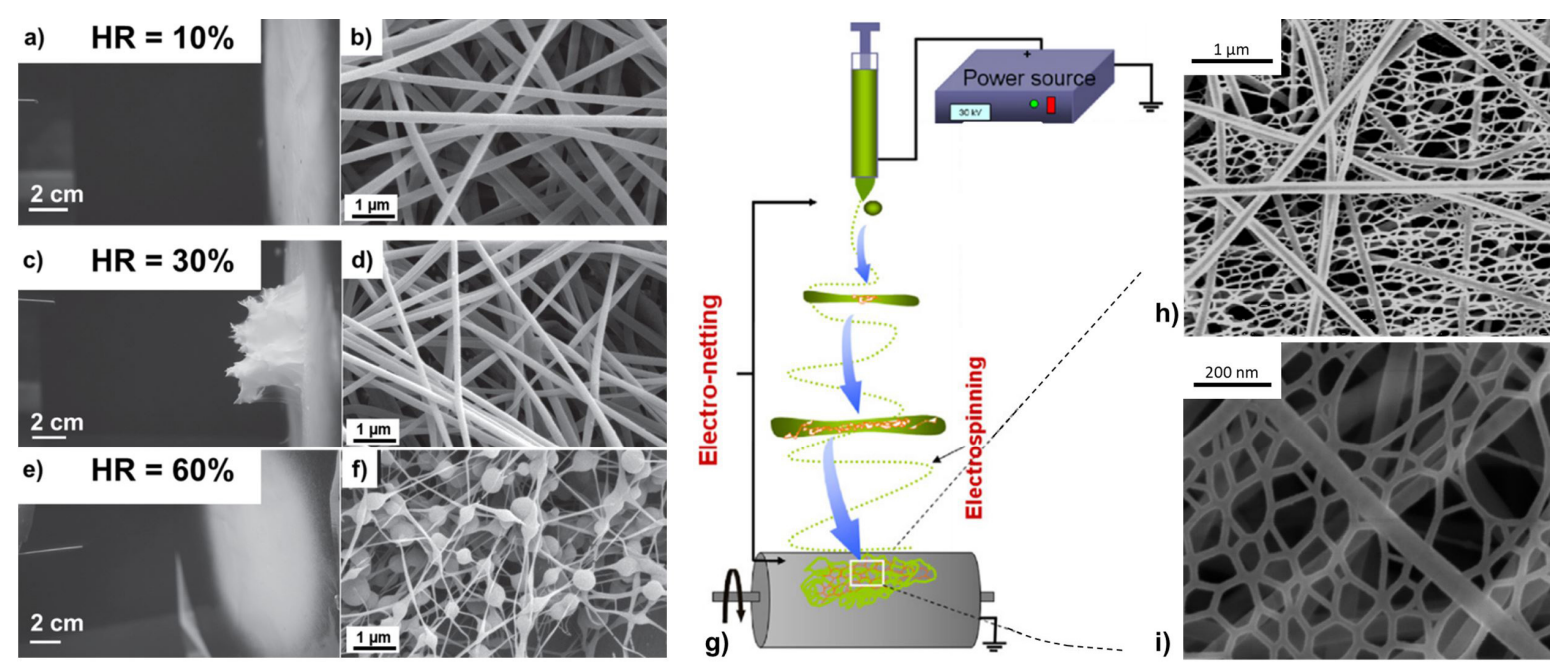

Figure 9. a-f) Effect of humidity on the formation of cotton-like structure after 40 minutes

of electrospinning of alginate solubilized in water for various relative humidity. a-c-e)

Pictures of the mat obtained at $\mathrm{RH}=10 \%, 30 \%$ and $60 \%$ respectively. b-d-f) SEM pictures of the corresponding fibers obtained at $\mathrm{RH}=10 \%, 30 \%$ and $60 \%$ respectively. a) to f) Reproduced with permission from. ${ }^{[137]}$ Copyright 2012, John Wiley and Sons. g) Mechanism of nanonet formation. Adapted with permission from. ${ }^{[141]}$ Copyright 2011 , Elsevier. h) Nylon 6 fibers and nanonets electrospun in formic acid at $25^{\circ} \mathrm{C}$ and $20 \% \mathrm{RH}$. Reproduced with permission from. ${ }^{[117]}$ Copyright 2006, IOP Publishing. i) Gelatin-NaCl fibers and nanonets electrospun in formic acid at $24^{\circ} \mathrm{C}$ and $25 \% \mathrm{RH}$. Reproduced with permission from. ${ }^{[141]}$ Copyright 2011, Elsevier.

\section{Specific electrospinning processes for dedicated controlled humidity and ambient conditions}

As humidity has been shown to play an important role on the structure of electrospun fibers and non-woven mats, the present chapter gives an overview of the developments of original electrospinning processes that exploit the ambient parameters and especially the level of humidity under specific controlled conditions, thus leading to new functional properties. 


\subsection{High-humidity and vapor induced electrospinning process}

Because water is able to induce phase separation between the components of the electrospun jet, researchers exacerbated this phenomenon by generating steam inside the electrospinning chamber (Figure 10a). Under such humid environment condition, it was shown that the electrospinning of a homogeneous solution of PAN and PVP solubilized in DMF resulted in the fabrication PANPVP core-shell fibers (Figure 10b) whereas uniform fibers were obtained when electrospinning was carried out at low $\mathrm{RH}=20 \% \cdot{ }^{[146]}$ A 1:4 PAN:PVP ratio was estimated in the fiber shell from XPS characterization indicating that indeed most of the PVP polymer was segregated on the fiber surface thanks to a vapor induced phase separation process.

It was also demonstrated that high-humidity electrospinning of PVDF solubilized in NMP (Figure 10c) allowed the preparation of membranes having an original "microsphere-fiber interpenetrated rough structure" (Figure 10d-f). ${ }^{[147]}$ The formation of such structure was explained by the combination of the low volatility and the high surface tension of the solvent and the high amount of water molecules in the air. On one hand, at low humidity, flat-cake and non-porous structures were obtained due to the very poor volatility of the solvent which prevents the efficient drying of the electrospun jet. Under high humidity however, phase separation can take place rapidly favoring the formation of the microsphere-fiber interpenetrated rough structure. On the other hand, when PVDF solubilized in DMF was electrospun, only fibers were formed due to the lower surface tension of DMF allowing the stretching of the electrospun jet while preventing any hydrodynamic instability responsible for bead formation. 

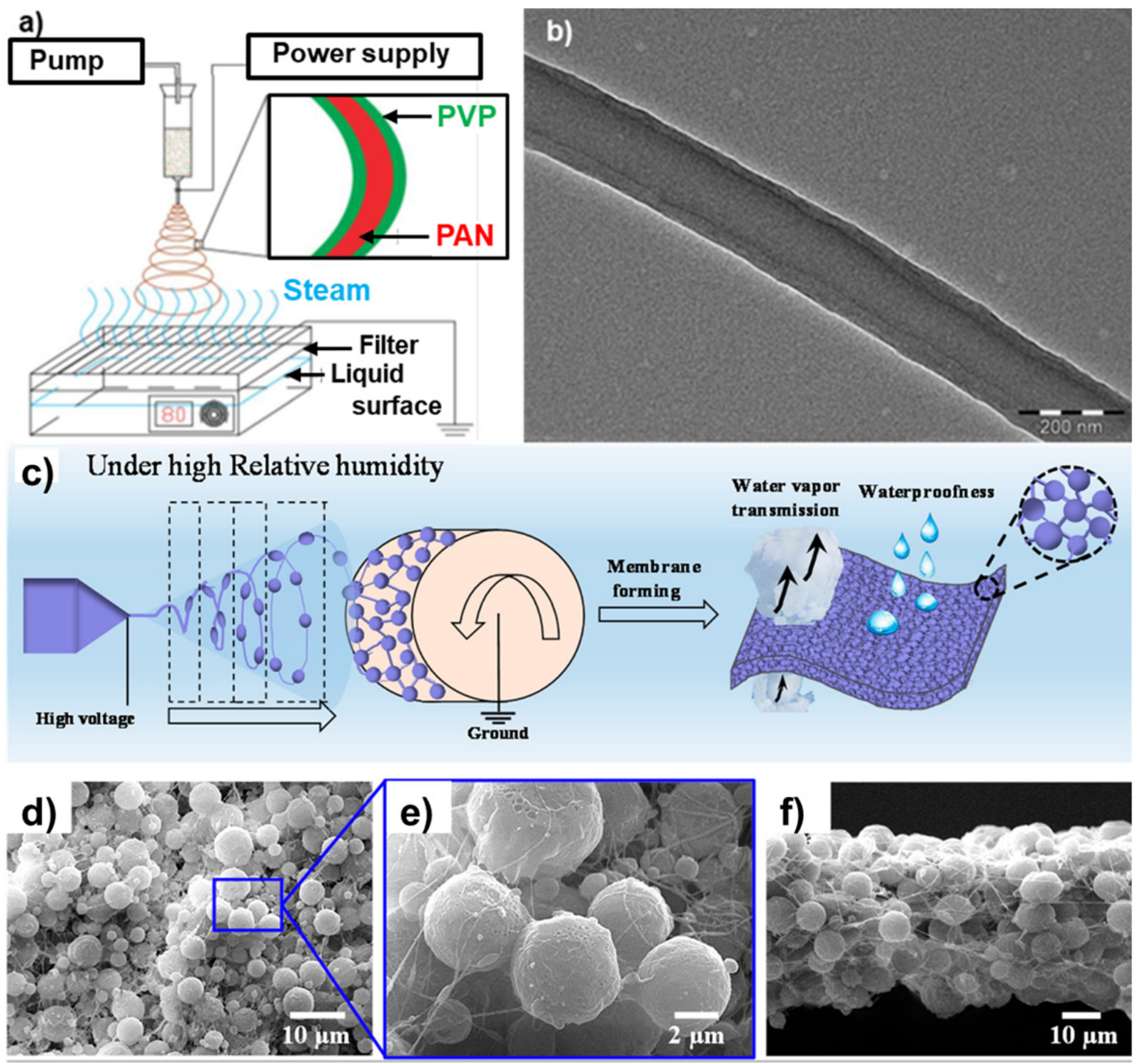

Figure 10. a) Steam generator introduced into the electrospinning setup for vapor induced phase separation. b) PAN-PVP core-shell fiber obtained by vapor induced phase separation from a 1:2 PAN:PVP homogeneous solution in DMF. a) and b) Reproduced with permission from. ${ }^{[146]}$ Copyright 2013, Royal Society of Chemistry. c) Electrospinning of PVDF under high humidity. d-e) SEM top view of the produced PVDF membrane. f) Cross-section of the PVDF membrane elaborated from high-humidity electrospinning. c) to f) Reproduced with permission from. ${ }^{[147]}$ Copyright 2018, American Chemical Society. 


\subsection{Low temperature and cryogenic electrospinning}

The fabrication of 3D cotton-like electrospun structures, as discussed in part 5.4, was optimized by Simonet et al. ${ }^{[148,149]}$ who proposed a process of electrospinning at controlled $\mathrm{RH}$ with a collector subjected to low temperature ranging between 200 and $220 \mathrm{~K}$. The authors applied this process, also called cryogenic electrospinning, for PLGA (Figure 11ab) as well as PEU. A minimum relative humidity of $30 \%$ at $200-220 \mathrm{~K}$ was necessary to supply enough vapor allowing to condensate in the form of ice crystals or water droplet. As void templates, these particles, trapped within the fibrous mesh, can thus easily be removed by lyophilization. An RH lower than $30 \%$ did not allow bringing enough water and classical dense fibrous meshes were obtained as in the case of standard electrospinning at room temperature. The same strategy was used by Leong et al. for the fabrication of PLA 3D cotton-like structures ${ }^{[150,151]}$ and by Sheikh et al. ${ }^{[152]}$ and Huang et al. ${ }^{[153]}$ in the case of the use of silk fibroin. The mechanism leading to the formation of these $3 \mathrm{D}$ cotton-like structures was proposed by Leong et al. ${ }^{[154]}$ and is, in fact, similar to the 3D honeycomb structuration obtained when electrospun fibers and electrosprayed particles are deposited simultaneously. ${ }^{[125,155]}$ Indeed, when ice crystals condensate at the surface of the collector, they form protuberances. Thus, when the jet enters in contact with the fibers and crystals already deposited, some strands are touching the crystals and others are hanging in air (Figure 11c). The hanged fiber strands that cannot release their charges form consequently repulsive domains whereas the portions of fibers in direct contact with the ice crystals release efficiently their charges towards the ground forming thus a highly attractive domain for the incoming jet which is landing. ${ }^{[125]}$ During the progress of the process, ice crystals are still deposited, insuring thus the building of a 3D multi-layered cotton-like structure. Similarly to what it is observed when electrospinning is carried out 
with micropatterned collectors, ${ }^{[124]}$ various $3 \mathrm{D}$ fibrous and porous structures are obtained as a function of the $\mathrm{RH}$ and the temperature due to their effect on the size and the density of crystals. Thus, the control of the balance between the rates of fiber production and ice crystal formation is paramount to tune the porosity and the average pore size.
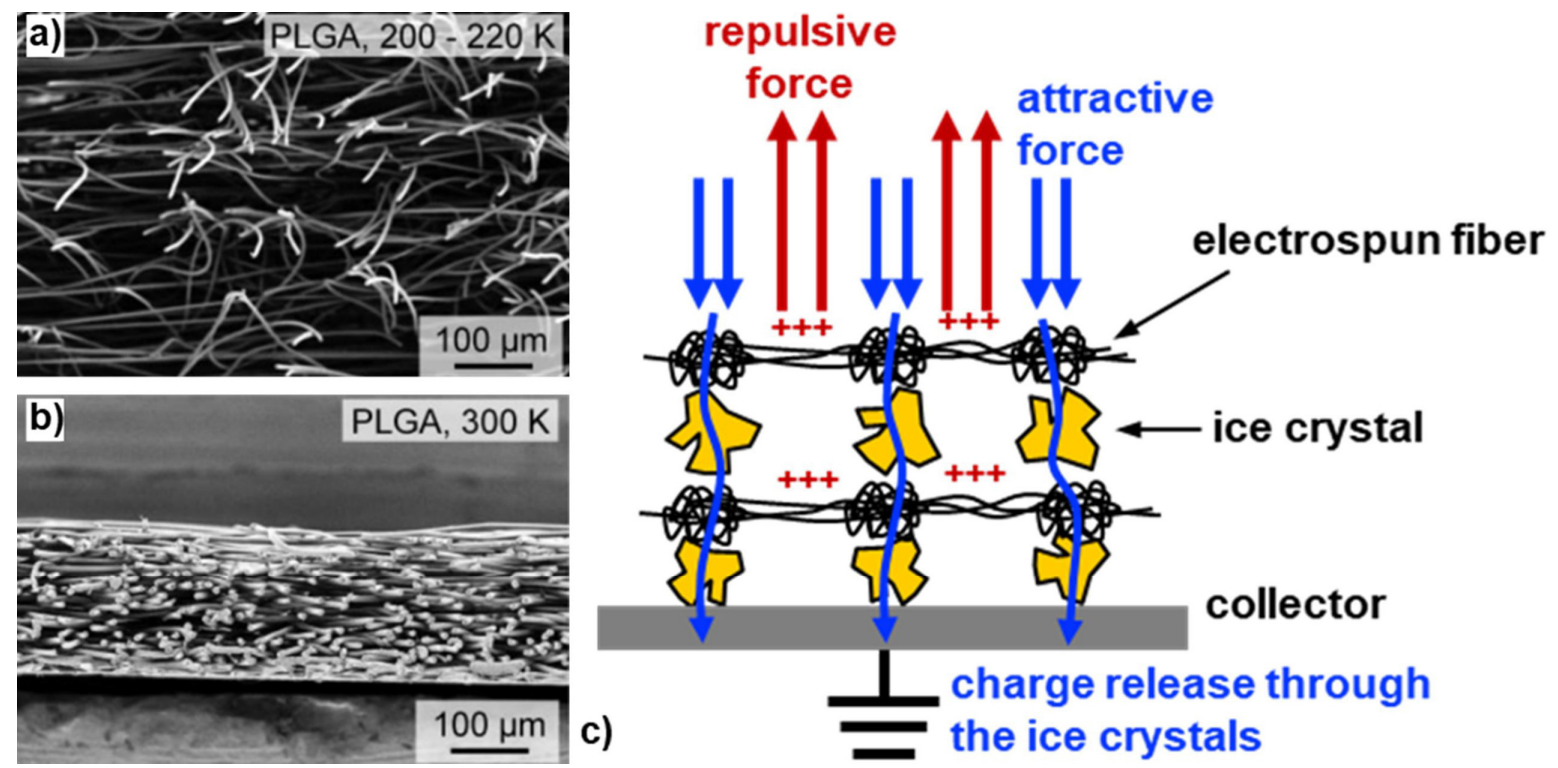

Figure 11. a) Cross-section of PLGA cotton-like scaffold obtained by low temperature electrospinning. b) Cross-section of PLGA scaffold obtained at room temperature electrospinning. a) and b) Reproduced with permission from. ${ }^{[148]}$ Copyright 2007, John Wiley and Sons. c) Mechanism of structuration during cryogenic electrospinning. The deposition of electrospun jet is guided by repulsive and attractive forces due to the charge strands of fibers hanging in air.

\subsection{Electrospinning onto insulating hydrophilic wet surfaces}

The deposition of electrospun fibers is generally carried out in an efficient manner when a grounded conducting collector is used. In this case, the electric charges brought by the jet can be released toward the ground, thus maintaining the electric field between the emitter and the collector and allowing the electrospun fibers to be deposited in a stable way over a 
long period of time. Conversely, when an insulating collector is used, the charges of the deposited fiber cannot be released to the ground. The charges remaining on the top surface of the deposited mat induce a force repulsing the incoming jet and preventing the efficient production of the non-woven mat. However, Choi et al. ${ }^{[156]}$ have shown that the control of humidity at the vicinity of the collector can be advantageously exploited in order to insure the deposition of electrospun fibers onto hydrophilic insulating substrates. Indeed, whereas no efficient electrospinning is experienced when a hydrophobic acrylic collector is used, the authors observed that the fibers are efficiently deposited when the collector is subjected to oxygen plasma treatment prior to electrospinning. Thanks to the plasma treatment rendering the collector surface hydrophilic, when electrospinning is carried out under high RH, adsorbed water molecules can act as an electrode layer allowing the charge release and resulting to the efficient fiber deposition. Moreover, using a mask, the collector surface was selectively modified under oxygen plasma treatment. Electrospinning on such hydrophilic/hydrophobic patterned collector under high local RH resulted to a structured fibrous deposition as observed when metallic collectors with grooves are used. ${ }^{[124]}$ Whereas aligned fibers are observed on hydrophobic dry areas, randomly deposited fibers are shown on the hydrophilic domains of the collector due to the adsorbed water molecules which favor the charge release to the ground.

\section{Applications for which the control of humidity is paramount}

As it was shown in the previous chapters, ambient humidity must be controlled during electrospinning in order to tune the structure of the fibrous membranes in all length scales. In a processing point of view, the control of RH is important in terms of processability ${ }^{[157]}$ 
and properties reproducibility of the fabricated materials. ${ }^{[35]}$ Obviously, as humidity has an impact on the fiber and mat morphologies, the physical properties are thus also linked with the RH imposed during electrospinning. As an example, Huang et $\mathrm{al}^{[38]}$ demonstrated the critical effect of $\mathrm{RH}$ on the overall mechanical properties (i.e. Young modulus, strain and stress at break...) of electrospun membranes elaborated either from PAN or PSU. In each of these cases, a sharp decrease of the Young modulus and the tensile strength by one order of magnitude was measured when RH increased from 10 to $40 \%$. This effect was corroborated with the cotton-like structure of the membranes obtained for the highest $\mathrm{RH}$ and inducing poor fiber-fiber interactions. The effect of $\mathrm{RH}$ imposed during electrospinning on the mechanical properties was also studied by Bandegi and Moghbeli ${ }^{[71]}$ who clearly observed a decrease of the tensile strength of SAN fibrous mats fabricated under high RH. In this case, the decrease of the mechanical properties of the mat was linked with the local internal porosity of the fibers induced through VIPS mechanism. Similar studies have also shown the effect of RH during electrospinning on the mat mechanical properties in the cases of PMMA, cellulose nanocrystal/PMMA ${ }^{[88]}$ and PAN. ${ }^{[158]}$ More recently, the mechanical properties were investigated at the level of individual PVDF nanofibers electrospun under various $\mathrm{RH}^{\left[{ }^{[159]}\right.}$ Much more ductile nanofibers were obtained at $60 \% \mathrm{RH}$ with a stiffness being four times lower and a strain at break four times larger than the corresponding values obtained for nanofibers electrospun at $30 \% \mathrm{RH}$. These local mechanical properties were corroborated with the huge difference in the fiber morphology: fibers having a wrinkled surface and internal pores were obtained at $60 \% \mathrm{RH}$ whereas smooth fibers with a monolithic solid core were obtained at $30 \% \mathrm{RH}$. Thus, all these results demonstrate the huge effect of RH on the mechanical properties measured at the different length scales from the individual fiber to the fibrous mat. 
Beyond the properties of the fibers and the mats, this chapter aims at presenting typical examples for which the precise control of humidity during electrospinning is of paramount importance to develop dedicated fibrous structures adapted for applications in various fields such as health, environment and energy.

\subsection{Scaffolds with optimized pore size for enhanced cell infiltration}

Electrospun membranes have been identified as good candidates to be used for biomedical applications such as tissue engineering for the repair of tissues or organs as for examples skin, ${ }^{[152]}$ bone, ${ }^{[22]}$ tendon $^{[25]}$ or heart. ${ }^{[160]}$ The fibrous structure of electrospun membranes allows the mimicking of the extracellular matrix favoring the promotion of cell adhesion, proliferation and differentiation as well as the nutrient transport and consequently the growth and repair of living tissues. Among the morphological properties that a biomaterials must endow to insure the $3 \mathrm{D}$ colonization by cells inside its volume, the pore size plays one of the most important role. When pores are too small, cells cannot penetrate efficiently inside the whole volume of the biomaterial whereas if the pores are too large, the resulting surface area of the biomaterials is too low to insure efficient interactions with cells. Thus, an ideal biomaterial should have an average pore size in the range of few tens to few hundreds of microns. ${ }^{[127]}$ However, conventional electrospinning leads to the fabrication of fibrous structures with an average pore size (i.e. the spacing between neighboring fibers) in micron range which is too small regarding the cell colonization issue. With this in mind, various strategies have been proposed to enlarge the average pore size such as post-treatment of the scaffold by ultrasonication, ${ }^{[138]}$ electrospinning in combination with electrospraying with the use of patterned collectors, ${ }^{[125]}$ fiber self- 
assembling ${ }^{[129]}$ electrospinning combined with a salt leaching technique. ${ }^{[161]}$ Interestingly, strategies allowing the production of cotton-like fluffy fibrous structures with large pores has been proposed thanks to the precise control of humidity during electrospinning at ambient or low temperature (LTE) (for the mechanisms, see respectively chapters 4.4 and $5.2)$.

On the basis of their preliminary results, ${ }^{[137]}$ Jeong et al. ${ }^{[138]}$ elaborated fluffy 3D scaffolds from RGD functionalized alginate, RGD (arginine glycine aspartic acid) being a peptide regulating cell adhesion. Compared to flat scaffolds (thickness $=(18 \pm 5) \mu \mathrm{m})$ obtained at $\mathrm{RH}=20 \%$, the presence of large pores within thick scaffolds (thickness $=(438$ $\pm 68) \mu \mathrm{m}$ ) prepared at $\mathrm{RH}=50 \%$ enhanced significantly primary human dermal fibroblast spreading, growth and infiltration. An ultrasonication post-processing step was also proposed for the samples obtained at $\mathrm{RH}=50 \%$ to get a scaffold thickness even greater which indeed provided more space for cell infiltration due to enlarged fiber-fiber distance. Using a sol-gel synthesis strategy, Norris et al. ${ }^{[162]}$ elaborated $\mathrm{SiO}_{2}-\mathrm{CaO}$ bioactive glass cotton-like fibrous scaffolds with several $\mathrm{CaO}$ amounts. The cotton-like structure of the scaffolds was achieved by electrospinning the silica precursors under $\mathrm{RH}$ higher than $50 \%$ followed by a thermal treatment to obtain the final 3D fibrous ceramic. It was shown that the introduction of $\mathrm{Ca}^{2+}$ in the precursor's solutions helps the formation of a $3 \mathrm{D}$ fibrous structure thanks to the separation of silicate and calcium ions in the electrospun jet causing more fiber-fiber repulsion during the deposition. The proposed 3D cotton-like scaffolds were further proposed for wound healing purposes.

LTE or cryogenic electrospinning have been the most used technique to fabricate $3 \mathrm{D}$ scaffolds with large pores thanks to a self-organization mechanism induced by the formation of ice crystals during the electrospun fiber deposition. ${ }^{[154]}$ Leong et al. ${ }^{[150]}$ and 
then Simonet et al. ${ }^{[149]}$ produced fluffy scaffolds with biopolyesters such as PLA and PCL for enhanced cell infiltration and even vascularization. Scaffolds prepared from LTE showed porosity in the range of $98-99.5 \%$ resulting to a decrease of the scaffold density by a factor of 10 compared to conventional electrospinning. These results were in-line with the 3 fold increase of average void size estimated from X-ray computed microtomography in the case of scaffolds having the thickest fibers (fiber diameter $\sim 9$ $\mu \mathrm{m})$. Human vena saphena cells (HVSC) were cultured during 7 days with PCL scaffolds. Whereas HVSC remained on the surface of scaffolds obtained from conventional electrospinning, efficient cell infiltration was observed within the scaffolds obtained from LTE. Similarly, Schneider et al. ${ }^{[163]}$ used LTE to elaborate cotton-like composite scaffolds made of PLGA and amorphous tricalcium phosphate (TCP). The authors demonstrated that such PLGA/TCP scaffolds exhibit excellent in vitro bone regeneration bioactivity. Furthermore, four weeks after implantation in calvarial defects in rabbits, a newly formed bone with spongeous morphology was observed. Such 3D cotton-like PLGA/TCP scaffolds seem to be ideal candidates for the repair of complex-shaped bone defects when the need of high mechanical strength in the early stage after implantation is of minor importance. Formica et al. ${ }^{[164]}$ elaborated PCL cotton-like scaffolds which were embedded in a sulfated alginate hydrogel with human chondrocytes to mimic the ECM of cartilage. While promoting the infiltration of cells, the fibrous nature of these PCL scaffolds also compensated the weak mechanical strength of the alginate hydrogel. Polysaccharides such as silk-based proteins have also been successfully processed by LTE by Bulysheva et al. $^{[165]}$. Whereas no fibroblast was observed within the thickness of scaffolds obtained from standard electrospinning, the very high porosity (93\%) associated with the large pore size of scaffolds obtained from LTE allowed an efficient cell infiltration. By adjusting the 
collector temperature during LTE, Huang et al. ${ }^{[153]}$ were able to tune the pore size of fluffy silk fibroin scaffolds. Whereas small pores were obtained from conventional electrospinning at room temperature, a collector temperature of $-80^{\circ} \mathrm{C}$ allowed the fabrication of scaffolds with medium pore size with an average value of $12 \mu \mathrm{m}$ and a larger average pore size of $37 \mu \mathrm{m}$ was achieved when the collector temperature has been set to $-50^{\circ} \mathrm{C}$. Mouse fibroblasts were cultured on the different scaffolds and it was clearly shown a much better cell infiltration in the depth of scaffolds having the largest pores. Sheikh et al. ${ }^{[152]}$ elaborated fluffy silk fibroin scaffolds within which human dermal fibroblasts were efficiently infiltrated. Human skin keratinocytes were even cocultured in a second step allowing the elaboration of bilayer skin substitute mimicking the dermal structure. Regarding biomedical applications, it must be noticed that ice crystals which induce the formation of large pores must be gently removed by an additional drying or freeze-drying step in order to maintain the 3D fibrous morphology. Nevertheless, LTE and cryogenic electrospinning are suitable techniques for biomedical applications as they do not introduce additional chemicals or toxic solvent in the process.

\subsection{Nanostructured fibers for sensor applications}

Electrospun membranes have been identified as adequate candidates for the elaboration of the active layer of sensors thanks to their high surface area. In order to further increase the surface exposed to the medium to be analyzed, Hwang et al. ${ }^{[166]}$ elaborated PMMA ultraporous fibers by electrospinning under high humidity $\mathrm{RH}=60 \%$. A single fiber was then transferred between two prongs of a quartz tuning fork (QTF) in order to measure the resonance frequency shift as a function of the amount of ethanol vapor in air. The authors 
demonstrated that the resonator elaborated with a PMMA ultraporous fiber was much more sensitive and faster than the one with a smooth fiber obtained from electrospinning at $\mathrm{RH}=30 \%$. Indeed, the ultraporous morphology of the fiber allows the rapid transport of large amount of ethanol inside the fiber inducing the decrease of its apparent modulus responsible of the resonance frequency shift. Kim et al. enhanced the sensitivity and the dynamic of this sensor by adding cellulose nanocrystal (CNC) in the fiber composition. ${ }^{[167]}$ Compared to pristine PMMA-based resonator, the addition of CNC has made it possible to preserve the ultraporous morphology of the fiber while increasing its apparent Young's modulus leading thus to the elaboration of faster and more sensitive sensors.

Another way to increase the surface area of a sensor is to decrease drastically the fiber diameter. In this perspective, electrospun membranes with the presence of nanonets should be excellent candidates when deposited on a quartz crystal microbalance to measure the mass variation by frequency shift due to analyte adsorption. Thus, depending on the electrospun polymer, it has been possible to selectively discriminate various molecules such formaldehyde in the case of poly(ethyleneimine) fibers, ${ }^{[26]} \mathrm{HCl}$ vapor for PANI functionalized PA6 ${ }^{[168]}$ and trimethylamine for PAA fibers. ${ }^{[169]}$ Nanonets ultrafine structures were also exploited for the colorimetric detection of L-ascorbic acid in the case of PANI-PA66 blend electrospinning. ${ }^{[170]}$ For this sensing application, PA66 is the structural matrix material whereas the color change of the fibers is due to a series of redox and doping reactions occurring between L-ascorbic acid and PANI. A limit of detection by naked eyes of L-ascorbic acid, as low as $50 \mathrm{ppb}$, was achieved. Such high sensitivity was ascribed to the nanonets that facilitate the rapid and efficient diffusion of the analyte in PANI. It is worth noting that the addition of PANI in the PA66-based solution favored 
advantageously the formation of nanonets thanks to high solvent evaporation rates and fast phase separations occurring at low humidity.

\subsection{Superhydrophobic fibrous materials for water repellency and remediation}

Superhydrophobic materials have paid attention as they can be used in environmental applications for the clean-up of oil and pollutants from water as well as for waterproof and breathable applications due to their water repellency properties. ${ }^{[171]}$ These materials are characterized by a water contact angle higher than $150^{\circ}$ and water droplets roll off the surface at low tilting angle $\left(\sim 5-10^{\circ}\right)$. Such behavior can be achieved by mimicking the surface morphology of lotus leaves which have the ability to trap air in the pores located on their rough surface maximizing thus the air/water droplet interface. ${ }^{[172]}$ It has been demonstrated that controlling the relative humidity during electrospinning can lead to the fabrication of fibrous materials having a hierarchical morphology due to the simultaneous presence of both inter-fibers voids and nanostructures at the scale of the fiber such as intra-porosity, surface wrinkles or beads-on-string leading thus to superhydrophobic behavior. ${ }^{[19]}$

Among the environmental applications, the selective adsorption of oil from water has been thoroughly studied over the past decades. Beyond the needed hierarchical structure, a good candidate for such applications needs optimal hydrophobic-oleophilic intrinsic properties, high buoyancy and high oil sorption in volume and rate. ${ }^{[173]}$ In this perspective, Lin et al. ${ }^{[19]}$ proposed the elaboration of PS electrospun membranes with fibers having a surface and internal porous structure when a low molar mass polymer was processed under moderate relative humidity of $40 \%$ due to efficient phase separation during the jet 
solidification (see Figure 5d). The optimal tuning of the polymer concentration $(30 \mathrm{wt} \%)$ and solvent weight ratio (THF:DMF 20:80) used for electrospinning led to fibers with a specific surface area (SSA) of $40 \mathrm{~m}^{2} / \mathrm{g}$ with a total pore volume of $0.24 \mathrm{~cm}^{3} / \mathrm{g}$ and an average pore width of $23 \mathrm{~nm}$. Oleophilic and hydrophobic properties of these PS membranes were evaluated and compared to a commercial PP non-woven dedicated for such application. A motor oil sorption capacity of $84 \mathrm{~g} / \mathrm{g}$ was measured for the PS hierarchically structured membrane which was three times larger than that of the commercial PP fabric. Nevertheless, these PS membranes suffer from poor mechanical and elastic properties due to their hierarchical porous structure preventing thus their recovering and reuse after squeezing for the recycling of adsorbed oil. In order to improve the overall mechanical properties, the same team proposed the elaboration by multi-nozzle electrospinning of composite membranes made of PS porous fibers for oil sorption capacity intertwined with PU fibers having excellent tensile strength and elongation. ${ }^{[64]}$ The relative humidity was optimized during electrospinning to achieve the highest PS fiber internal porosity. As a result, an SSA of $50 \mathrm{~m}^{2} / \mathrm{g}$ was measured when electrospinning was conducted with an $\mathrm{RH}=45 \%$ whereas an SSA of only $4 \mathrm{~m}^{2} / \mathrm{g}$ was achieved for $\mathrm{RH}=$ $20 \%$. However, although an increase of the SSA by a factor 12 was measured when increasing the $\mathrm{RH}$ from $20 \%$ to $45 \%$, an increase of the oil sorption capacity by a factor 2 was only achieved. This result demonstrates that the inter-voids play a predominant role rather than intra-porosity. The introduction of intertwined PU fibers within the fibrous mesh not only enhanced the mechanical properties but also allowed the tuning of the intervoids distance. The sorption capacity of the composite PS/PU membranes was characterized after several cycles of squeezing for oil recovering showing their potential application for water remediation. Tang et al. also elaborated PS ultraporous fibers for oil 
removal from water. ${ }^{[174]}$ They proposed to use camphene and tetraethoxysilane (TEOS) as porogen agents in order to enhance the pore formation induced under high humidity. Thanks to the combination of water VIPS, TEOS diffusion towards the surface of the electrospun jet and camphene accumulation in the jet core, fibers having a highly hierarchical porous structure are obtained allowing a maximum oil sorption capacity of $105 \mathrm{~g} / \mathrm{g}$. Hierarchically structured membranes embedding intra-porous fibers were also developed with other kinds of hydrophobic-oleophilic polymers for water remediation applications. As an example, Zaarour et al. ${ }^{[97]}$ obtained PVDF macro-porous fibers when acetone was used as the solvent during electrospinning under high RH due to TIPS and VIPS mechanisms (see Figure 6C-F). A sorption capacity of $50 \mathrm{~g} / \mathrm{g}$ was achieved in the case of silicon oil and $38 \mathrm{~g} / \mathrm{g}$ for motor oil. Bandegi and Moghbeli ${ }^{[71]}$ elaborated ultraporous SAN fibers who obtained the highest internal fiber porosity at the highest studied relative humidity (i.e. $\mathrm{RH}=60 \%$ ) due to efficient water VIPS. Under these conditions a motor oil sorption capacity of $160 \mathrm{~g} / \mathrm{g}$ was measured. It is however worth noting that the comparison of sorption capacities must be discussed with special care because it depends on the oil nature which was not mentioned in the cited articles.

Different kinds of hierarchically structured fibers having superhydrophobic properties were also elaborated for waterproof and breathable applications. Paulus et al. elaborated ultraporous fibers from poly(alkyl-p-xylylene) (PPX), a polymer known for its excellent mechanical, biocompatible and biostable properties. ${ }^{[175]}$ Three series of polymers with propyl, butyl or heptyl lateral alkyl substituents were synthesized and subsequently electrospun under various RH. Whereas smooth fibers were obtained at low $\mathrm{RH} \leq 25 \%$, increasing the RH up to $60 \%$ led to the formation of pores with increasing density. At the highest studied $\mathrm{RH}$ above $80 \%$, the pores merged together forming larger non-circular 
cavities. The authors also electrospun the three kinds of PPX solubilized in THF at low concentration resulting in the formation of beads-on-string structures. Under such conditions, the beads, having a characteristic size in the order of $10 \mu \mathrm{m}$ were covered by a high density of pores when electrospun at high $\mathrm{RH}$ while the fibers of diameter ranging between $100 \mathrm{~nm}$ and $1 \mu \mathrm{m}$ showed a wrinkled or smooth surface. These porous beads-onstring morphology being highly hierarchically structured was consequently superhydrophobic with a maximum contact angle of $160^{\circ}$ in the case of poly(butyl-pxylylene) and poly(heptyl-p-xylylene) mats electrospun from THF at RH $>75 \%$. Furthermore, low roll-off angles of $10-15^{\circ}$ were also measured allowing water droplets to roll and pick up dirt or dust particles from the hierarchically structured surface making these materials excellent candidates for self-cleaning applications. $\mathrm{Li}$ et al. ${ }^{[176]}$ demonstrated that polyurethane/fluorinated polyurethane (PU/FPU) fibrous membranes with both good waterproof, breathable as well as mechanical properties can be obtained. In this case, fibers didn't present pores on their surface or in their core. An optimal interfiber porous morphology can be simply achieved under controlled RH during electrospinning. The authors showed a significant increase of the porosity and the contact angle with RH (Figure 12) whereas only a slight increase of the mean pore size and even no evolution of the maximum pore size were measured. Indeed, the increasing of $\mathrm{RH}$ favored a rapid and efficient solidification of the PU/FPU electrospun jet leading to poor adhesion at the fiber-fiber contact points. Thus, membranes obtained at high $\mathrm{RH}=60 \%$ were much more fluffy than those obtained at lower RH. The slight increase of the mean pore size with RH may be ascribed to the increase of the fiber diameter induced by the efficient jet solidification at high humidity. On one hand, the breathable property is linked to the water vapor transmission capacity of the membrane which is favored by a high 
porosity and a large mean pore size. On the other hand, the waterproof property is linked to the maximal hydrostatic pressure for which no water penetration is observed across the membrane and which is favored by a high contact angle. Thus, membranes obtained at the highest RH were the best candidates for both breathable and waterproof applications. An optimization of the membrane thickness by simply tuning the electrospinning time allowed finally the elaboration of membranes with mechanical properties adapted for such applications. More recently, Jiang et al. ${ }^{[147]}$ elaborated PVDF membranes for waterproof and breathable applications. An original membrane structure with a very high density of microparticles was obtained when electrospinning was carried out under a very high amount of humidity (see $\S 5.1$ and Figure 10). These microsphere-fiber interpenetrated rough membranes were thus superhydrophobic and showed excellent water repellency properties making them good candidates for waterproof and breathable applications.
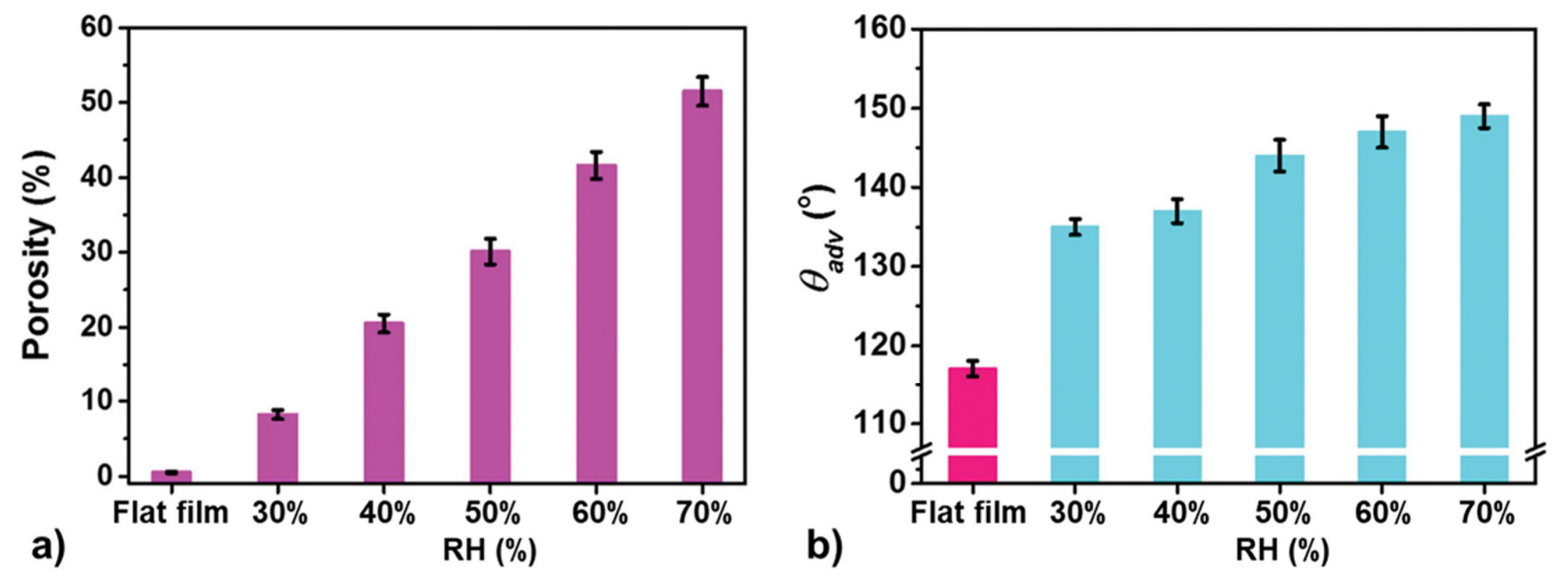

Figure 12. Effect of relative humidity during electrospinning of polyurethane/fluorinated polyurethane (PU/FPU) on a) the porosity and b) on the water contact angle of the membranes. a) and b) Adapted with permission from. ${ }^{[176]}$ Copyright 2016, John Wiley and Sons. 


\subsection{Inorganic fibers for applications in catalysis and energy}

It was demonstrated that hierarchically structured inorganic composite materials obtained from template polymeric electrospun membrane can be excellent candidates for catalytic applications due to their high surface area with both nano-, micro- and meso-pores. Among them, carbon fibers obtained from the carbonization of electrospun PAN membranes have shown excellent catalytic activity. For example, the high amount of nitrogen atoms remaining in the carbon structure after PAN carbonization allowed the use of such materials as metal-free catalyst in the catalytic oxidation of $\mathrm{H}_{2} \mathrm{~S}$ to sulfur ${ }^{[177]}$ or for the steam- and oxygen-free catalytic dehydrogenation of ethylbenzene to styrene. ${ }^{[178]}$ Furthermore, thanks to the presence of both macro-pores limiting the diffusive resistance to mass transport and micro- and/or meso-pores enhancing the dispersion of active sites, hierarchical porous carbons are used as highly efficient supports for various metal catalysts. ${ }^{[179]}$ For example, Co nanoparticles can be loaded during the carbonization step of electrospun PAN fibers to be used for the Fischer-Tropsch process for the conversion of synthesis gas into clean synthetic hydrocarbons. ${ }^{[180]}$ As for PAN, poly(imide) (PI) can also be used as a precursor for the fabrication of carbon fibers with nitrogen atoms remaining in the carbon structure after its carbonization. In this case, Tian et al. elaborated, from a three steps process (Figure 13a), $\mathrm{Co} / \mathrm{Co}_{3} \mathrm{O}_{4} @ \mathrm{HPCNF}$ carbon nanofibers having a hierarchical porous structure and embedding $\mathrm{Co} / \mathrm{Co}_{3} \mathrm{O}_{4}$ noble-metalfree hetero nanoparticles for oxygen reduction/evolution electrocatalytic reactions occurring during the charging and discharging processes of rechargeable batteries. ${ }^{[72]}$ In the first step of the process, PI nanofibers were fabricated by electrospinning under various RH leading to ultraporous fibers having a porous density increasing with the humidity. Then, the PI nanofibers were immersed in a cobalt acetate solution and further 
subjected to hydrothermal reaction allowing the growth of $\mathrm{Co}_{3} \mathrm{O}_{4}$ nanoparticles on their surface. A carbonization step under inert atmosphere ended the process and led to $\mathrm{Co} / \mathrm{Co}_{3} \mathrm{O}_{4} @ \mathrm{HPCNF}$ fibers with a hierarchical porous carbon structure decorated by $\mathrm{Co} / \mathrm{Co}_{3} \mathrm{O}_{4}$ nanoparticles (Figure 13b-e). During this last carbonization step, meso-pores and large cavities are formed through the collapse of adjacent primary pores in the PI fibers. $\mathrm{Co} / \mathrm{Co}_{3} \mathrm{O}_{4} @ \mathrm{HPCNF}$ fibers with large cavities having a hierarchical porous structure were thus obtained from PI fibers obtained at $\mathrm{RH} \geq 50 \%$. The calculated Brunauer-Emmett-Teller (BET) surface area calculated from nitrogen sorption experiments (Figure 13f) demonstrated the presence of meso-pores with an average size of 4-70 $\mathrm{nm}$ increasing with the density of primary pores of diameter in the range of 4-5 $\mathrm{nm}$. Such strategy presents the advantage to elaborate hierarchical porous carbon fibers from a template-free strategy. Thanks to this hierarchical morphology, the authors demonstrated a remarkable electrocatalytic activity of the $\mathrm{Co} / \mathrm{Co}_{3} \mathrm{O}_{4} @ \mathrm{HPCNF}$ fibers obtained from PI electrospun at $\mathrm{RH}=50 \%$ for both oxygen reduction reaction (ORR) and oxygen evolution reaction (OER) (Figure 13g-h). Such result was the consequence of the presence of $\mathrm{Co} / \mathrm{Co}_{3} \mathrm{O}_{4}$ nanoparticles and nitrogen atoms in the carbon structure and the unique hierarchical porous carbon structure of the fibers.

Aghasiloo et al. ${ }^{[181]}$ took advantage of the control of $\mathrm{RH}$ during electrospinning in order to fabricate $\mathrm{TiO}_{2}$ nanofibers with optimized properties for photocatalytic applications. These nanofibers, obtained from the calcination of PVP/Titanium (IV)isopropoxide (TTIP) electrospun precursor, have shown an increase by a factor 2.6 of the BET surface when electrospinning was conducted under an $\mathrm{RH}$ of $65 \%$ instead of $50 \%$. Although the mechanism behind this effect was not clarified, this increase in BET surface area was 
related with a remarkable improvement of the photocatalytic activity characterized by the UV-light degradation of methylene blue in aqueous suspension.
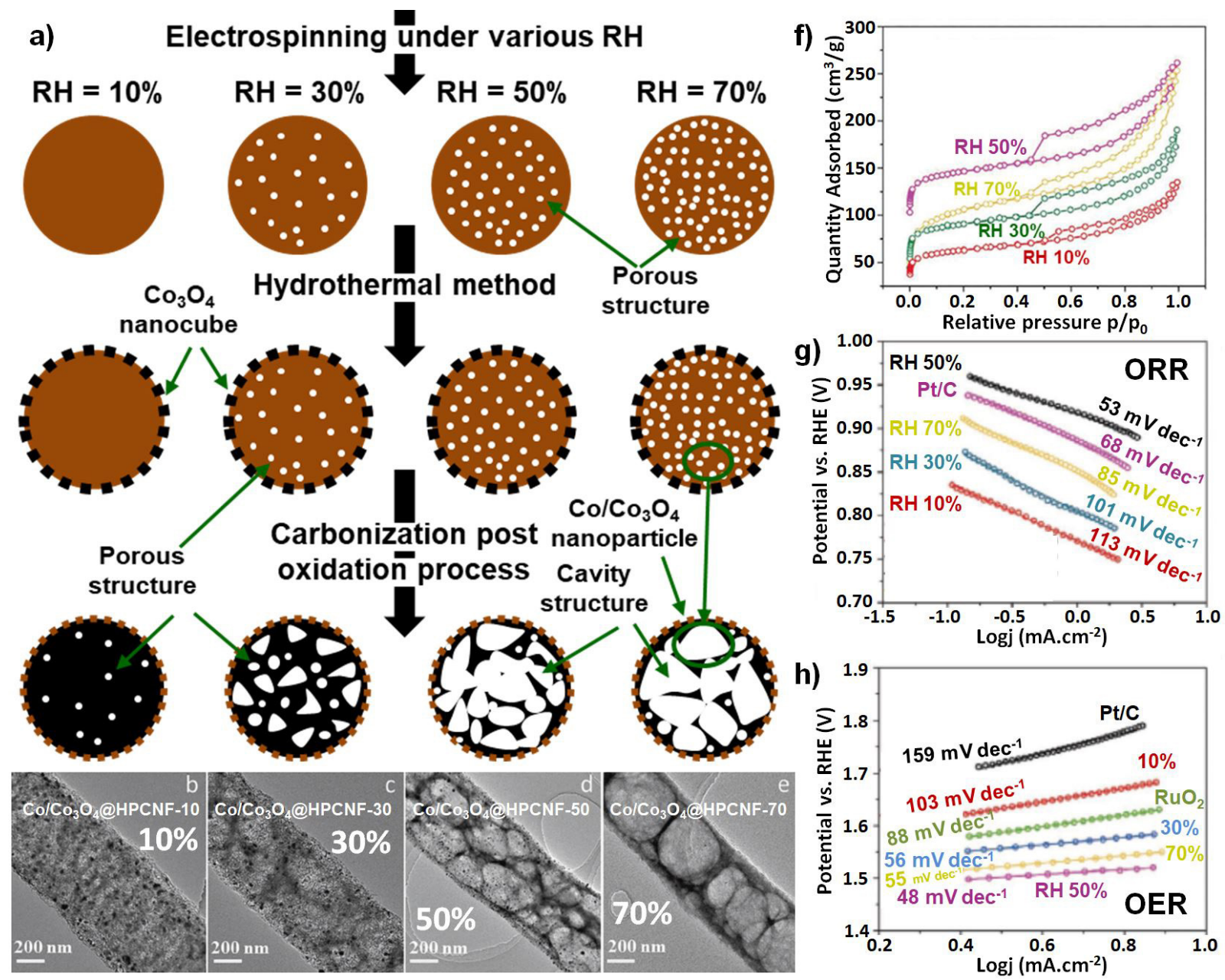

Figure 13. a) The three steps to obtain hierarchical porous carbon nanofibers embedding $\mathrm{Co} / \mathrm{Co}_{3} \mathrm{O}_{4}$ hetero nanoparticles $\left(\mathrm{Co} / \mathrm{Co}_{3} \mathrm{O}_{4} @ H P C N F\right)$. TEM of $\mathrm{Co} / \mathrm{Co}_{3} \mathrm{O}_{4} @ H P C N F$ obtained after hydrothermal and carbonization steps of nanofibers electrospun at b) $\mathrm{RH}=$ $10 \%$, b) $\mathrm{RH}=30 \%$, b) $\mathrm{RH}=50 \%$, b) $\mathrm{RH}=70 \%$. f) Nitrogen adsorption-desorption isotherms of $\left.\mathrm{Co} / \mathrm{Co}_{3} \mathrm{O}_{4} @ \mathrm{HPCNF} . \mathrm{g}\right)$ Tafel plots of Co/Co3O4@HPCNF and Pt/C obtained from ORR polarization curves. h) Tafel plots of Co/Co3O4@HPCNF, Pt/C and 
$\mathrm{RuO}_{2}$ obtained from OER polarization curves. a) to h) Adapted with permission from. ${ }^{[72]}$ Copyright 2020, John Wiley and Sons.

\subsection{Nanofibrous mats for air filtration}

Air pollution by particulate matter (PM) due to human activities is causing very important health and environmental issues. The elaboration of systems capable to filter pollutants as small as $\mathrm{PM}_{1}$ regrouping particles of aerodynamic diameter lower than $1 \mu \mathrm{m}$ is crucial as they are the most dangerous. ${ }^{[182]}$ In order to be efficient, a filtering system needs to trap small pollutants while facilitating the air flux preventing high pressure drop across its thickness and rapid filter clogging. The mechanisms involved in trapping PM in a fibrous filter are size-exclusion, inertial impaction, interception and Brownian diffusion. Electrostatic attraction of PM may also occur in the case of filters previously subjected to electret treatment. ${ }^{[183]}$ Fibrous membranes elaborated via electrospinning have been identified as excellent candidates for this purpose thanks to their high surface area-tovolume ratio as well as possible in-situ charging. ${ }^{[35,183,184]}$ In order to ensure high filtration efficiency while preventing airflow resistance, Cai et al. elaborated 3D fluffy PS microfibrous membranes. ${ }^{[184]}$ By adjusting the RH during electrospinning, they were able to tune the fiber diameter, the packing density of the fibers as well as the amount of embedded charges. Thus, an optimal RH of $60 \%$ was found resulting to both high filtration efficiency and low pressure drop (Figure 14a). PS/PAN/PS filters, made of a thin layer of PAN nanofibers in sandwich between two layers of PS microfibers, were finally built. Such optimized electret filters took advantage of the large electric resistivity and high porosity of PS fluffy microfibers and the high surface area-to-volume ratio and high 
polarity of PAN nanofibers. Similar results were obtained by Wang et al. for PLA-based filters made of interlaced or multilayer microfibers and nanofibers. ${ }^{[91-93]}$

The improvement of the filtration efficiency requires the fabrication of membranes with the finest possible fibers while maintaining high mechanical properties. In order to meet such requirements, Zhang et al. proposed the elaboration of robust PMIA membranes which embedded a nanonet structure when electrospinning was carried out at low humidity $\mathrm{RH} \leq 25 \% .{ }^{[133]}$ These membranes exhibited very high filtration efficiency mostly by sieving mechanism nevertheless with a relatively high pressure drop. Recently, the same group elaborated PAN membranes combining both nanonets for improved filtration and a fluffy structure for low pressure drop (Figure 14b-e). ${ }^{[185]}$ Such dual fibrous structure, showing a very low packing density, was obtained thanks to the addition of tetrabutylammonium chloride (TBAC), a cationic surfactant, and the control of humidity during electrospinning allowing the formation of nanonet structures homogeneously distributed in the whole volume of the membrane. More precisely, the addition of TBAC may induce ion-dipole interactions between the cationic group of TBAC and the polar nitrile group of PAN resulting to the release of free chloride ions of TBAC which enhances the polarization/charging of the electrospun jet. This high level of charges allows the efficient production of charged droplets simultaneously with the primary electrospun jet. During their travel in air, the shape of the charged droplets moves from sphere to film. During this step, the film may be subjected to phase separation due to solvent evaporation leading the final deposition of solid 2D films, with some defective pores, attached between the fibers. When the $\mathrm{RH}$ is increased from $15 \%$ to $35 \%$, the $2 \mathrm{D}$ films turned into nanonets, i.e. networks of ultrafine nanowires of $\sim 20 \mathrm{~nm}$ in diameter (Figure 14b). A further increase of the RH from $35 \%$ to $45 \%$ led to the decrease of the 
coverage of nanonets with a total disappearance when electrospinning was carried out under $\mathrm{RH} \geq 55 \%$. Thus, as explained through numerical simulations, the membranes elaborated under $\mathrm{RH}=35 \%$ exhibited high filtration efficiency, mostly by a sieving mechanism, thanks to the high coverage ratio of nanonets, while limiting the pressure drop thanks to the fluffy fibrous structure (Figure 14d-e).
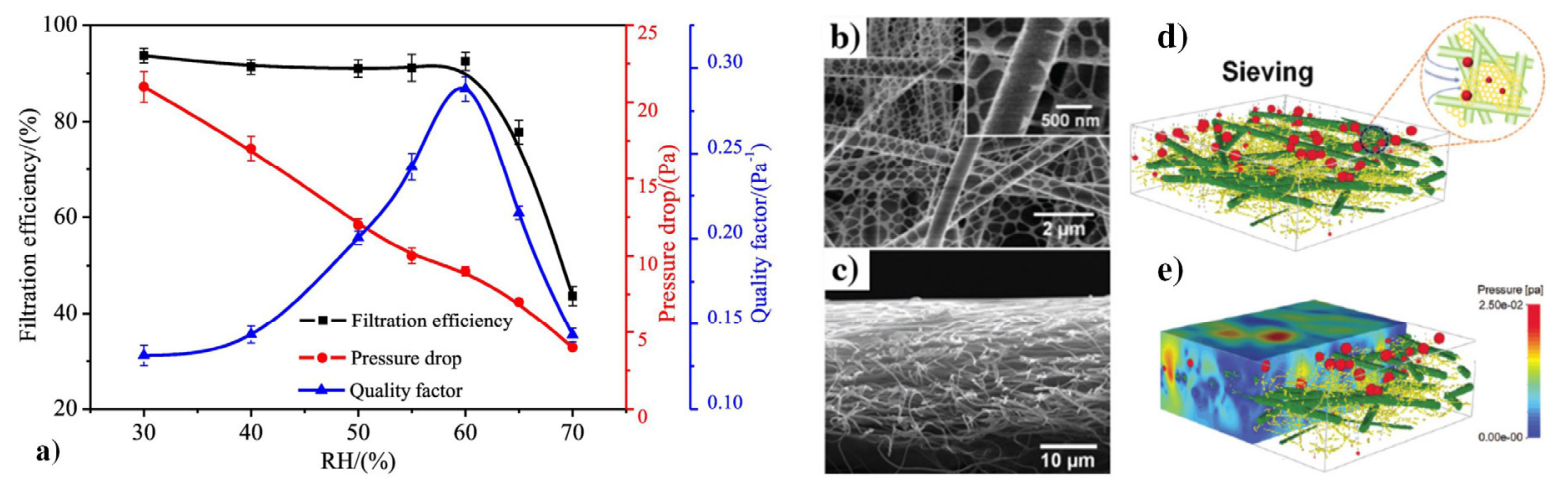

Figure 14. a) Filtration efficiency for $0.3 \mu \mathrm{m}$ particles $\left(\mathrm{PM}_{0.3}\right)$, pressure drop measured at $5.3 \mathrm{~cm} / \mathrm{s}$ and quality factor of PS filters. Reproduced with permission from. ${ }^{[184]}$ Copyright 2020, Elsevier. b) SEM top-view and c) cross-section of fluffy dual fibrous network of PAN nanofibers and nanonets. d) and e) Modeling of airflow and $\mathrm{PM}_{0.3}$ trapping by sieving in a nanofiber/nanonet structure. b) to e) Reproduced with permission from. ${ }^{[185]}$ Copyright 2019, John Wiley and Sons.

\section{Summary and outlook}

It has been shown that humidity plays a key role in controlling the fiber morphology and the structure of electrospun nonwovens as it influences the evaporation rate of solvents and the solidification rate of fibers. Whether the solidification of the electrospun fibers occurs rapidly or slowly, the morphology of the fibers and the structure of the mats may change depending on the properties of the processed formulation. Figure 15 summarizes 
how humidity influences the solidification rate differently according to both the solvent (aqueous, hydrophilic or hydrophobic) and the polymer (hydrophilic, hygroscopic or hydrophobic). For hydrophilic and hygroscopic polymers solubilized in aqueous or hydrophilic solvents, the increase of humidity delays the solidification of the fibers due to water absorption and polymer plasticizing. Consequently, fibers are coarser at low humidity than at high humidity. Besides, high humidity favors the apparition of bead-onstring fibers and films. In the case of hydrophobic polymers, the increase of humidity usually shortens the solidification time of the fibers. Consequently, low humidity leads to thin fibers while in contrast high humidity yields coarser fibers, and possibly porous fibers, cotton-like structure and nanonets. Furthermore, fiber orientation can disappear at high humidity due to jet discharge. Thus, for a given composition of a solution to be electrospun, choosing the RH (at a given temperature) is a way to tailor the structure and/or the morphology of the final material at the length scales ranging from the nonwoven mat to the fiber structure itself and even down to the crystalline structure of the polymer leading thus to the control of various properties of the mats such as their mechanical strength, biological properties (controlled release rates, cell infiltration), catalytic properties, hydrophobic properties and so on. 

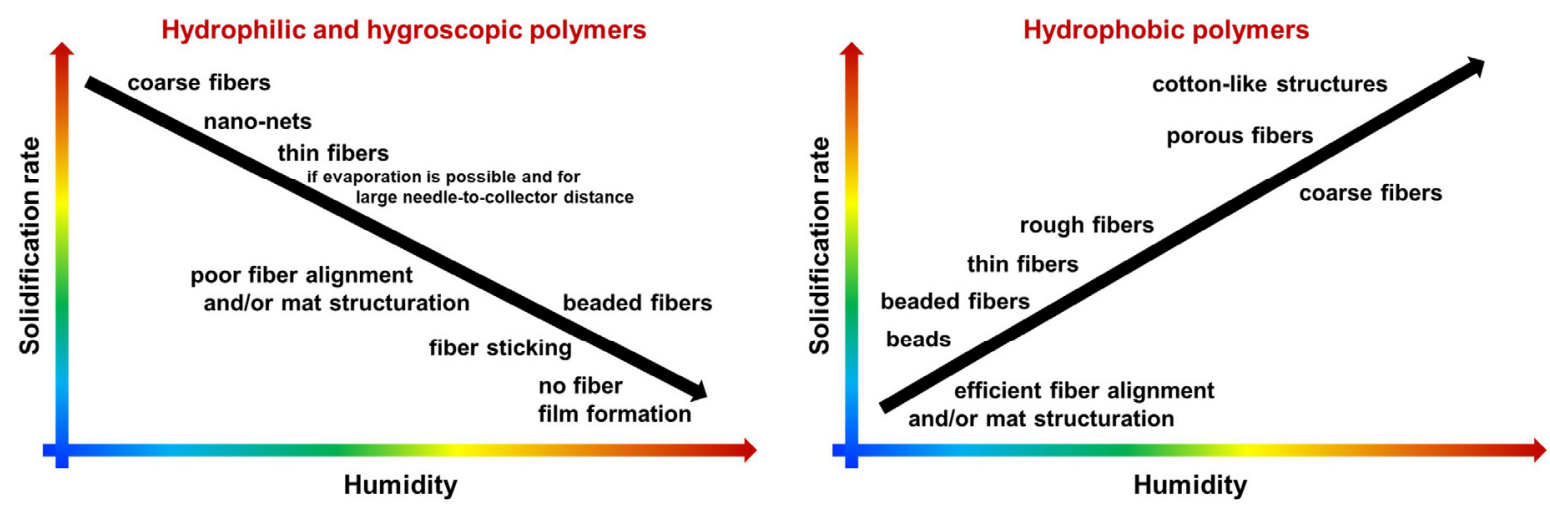

Figure 15. Electrospun nanofiber morphologies and mat structures as a function of humidity, solidification rate and polymer hydrophilicity.

It is anticipated that more complex fibrous structures could be designed with improved properties and/or to target new applications through the fine tuning of the interactions between the air and the processed molecules. The control of these interactions can be considered thanks to i) the development of specific electrospinning processes allowing the production under dedicated air environments and ii) the processing of dedicated polymer/solvents/additives systems.

Possible developments via the processing under dedicated air environments. The design of the entire electrospinning process must be thought out in order to have a fine tuning of the ambient humidity. For instance, by simply varying the properties of air (i.e. humidity and temperature) during the time of production, it is possible to consider the elaboration of fibrous mats having a controlled multilayer, sandwich or gradient structure through their thickness. Specific emitters, similar of that used in blowspinning ${ }^{[186]}$ while imposing a much lower air flow rate, could be designed allowing the delivering of air around the vicinity of the Taylor cone with controlled mass flow rate, humidity and temperature. Elsewhere, for the bending region and in the whole electrospinning chamber another set of temperature and humidity could be imposed for ambient air. Such processing fine tuning 
would allow the jet to be emitted under stable conditions while favoring the structuration of the fiber and/or the mat during the whipping movement until the hitting of the collector. In the case of multiple electrospinning for the fabrication of fibrous composites embedding different kinds of intertwined fibers, the chamber of electrospinning could be designed such as each fiber is produced under its specific air environment. Indeed, using a rotating drum as collector, the electrospinning device can be easily segmented in two or three independent areas allowing the simultaneous deposition of fibers having their own nano-micro structure. Finally, it is also worth noting that needleless electrospinning processes dedicated to industrial production rates must be designed with special care taking into account the high level of interactions between the free surface of the polymer solution at the vicinity of the emission area and the surrounding air. In the humidity point of view, the design of needleless emitters should allow to limit the exchanges with air and reduce the residence time of the polymer solution on the free-surface of needleless emitters. Needleless emitters delivering a controlled air environment near the jets emission area can also be envisaged.

\section{Possible developments via the processing of dedicated polymer/solvents/additives}

systems. As the structure of the fiber and/or the mat is induced by the interactions between the electrospun jet and the air, the fine tuning of the polymer/solvents/additives formulation composition is of paramount importance. As the hydrophobicity/hydrophilicity of the processed polymer plays a major role on the interactions with the water molecules in air, its modification by the grafting of specific side groups could be a way to adjust these interactions. However, these modifications must be carried out with special care without altering the final properties of the polymer which could be detrimental to the intended application. The adjustment or choice of the 
solvent is another way to better target specific fiber structure and more research needs to be done in this area. In general, the solvent is mainly chosen in relation with its capacity to solubilize the polymer and to adjust the overall volatility of the formulation to be electrospun. However, as it was discussed in the present review, the solvent plays a major role on the structure of the fibers at the different length scales. Thus, its choice has also to be thought as a function of the fiber structure which is needed for the application. As an example, ternary and/or more complex formulations may be considered for humidityinduced nanofiber structuration. As shown in chapter 3.2, water can act as a ternary nonsolvent which may induce phase separation during solvent evaporation leading to ultraporous fibers when hydrophobic polymers are electrospun. The affinity of the solvent with humidity and its vapor pressure are key parameters for the control of the porous morphology. Thus, the choice of the solvent and even more, the choice of a secondary solvent have to be thought with special care and in regards with its affinity with humidity when fibers having secondary morphologies are targeted. In order to enhance or modify the interactions between the electrospun jet and humidity, dedicated additive may also be introduce in the formulation. Such additives could be used as template molecules, porogen or could directly react with the water molecules present in air. The addition of ions in the solution could favor the formation of nanonets or fiber branching.

In summary, the control of humidity during electrospinning is mandatory to control the fiber structure at all length scales to target a wide range of applications. The deep understanding of the effect of humidity on the fiber structure will help the researchers to better control the fiber structure or even more to design new kinds of structures. Finally, in terms of process repeatability, the control of humidity must thus be implemented in all electrospinning devices: from lab-scale up to industrial production. 


\section{Abbreviations}

\begin{tabular}{ll}
\hline Solvents & \\
DCM & dichloromethane \\
DMAc & N,N-dimethylacetamide \\
DMF & N,N-dimethylformamide \\
Mc & methylene chloride \\
NMP & N-methyl-2-pyrrolidone \\
THF & tetrahydrofuran \\
HFIP & Hexafluoroisopropanol \\
& \\
\hline Polymers & \\
CA & cellulose acetate \\
PA & polyamide \\
PAA & poly(acrylic acid) \\
PAN & poly(acrylonitrile) \\
PANI & polyaniline \\
PCL & poly(c-caprolactone) \\
PEG & poly(ethylene glycol) \\
PEO & poly(ethylene oxide) \\
PEI & poly(ether imide) \\
PLA & poly(L-lactide) \\
PLGA & poly(y(lactide-co-glycolide) \\
PS & polystyrene \\
PSU & polysulfone \\
PU & polyurethane \\
PVA & poly(vinyl alcohol) \\
PVP & poly(vinyl pyrrolidone) \\
SAN & poly(styrene-co-acrylonitrile) \\
PPV & Poly(p-phenylenevinylene) \\
PNIPAM & poly(N-isopropylacrylamide) \\
PVDF & poly(vinylidene fluoride) \\
PEU & poly(ester-urethane) \\
PMIA & Poly(m-phenylene isophthalamide) \\
&
\end{tabular}

\section{Acknowledgments}

This work was supported by the Région Alsace through the FUI-16 CLARIFIL Project. 


\section{References}

[1] Z. M. Huang, Y. Z. Zhang, M. Kotaki, S. Ramakrishna, Compos. Sci. Technol. 2003, 63, 2223.

[2] A. Greiner, J. H. Wendorff, Angewandte Chemie International Edition 2007, 46, 5670.

[3] A. Haider, S. Haider, I.-K. Kang, Arabian Journal of Chemistry 2018, 11, 1165.

[4] J. Xue, T. Wu, Y. Dai, Y. Xia, Chemical Reviews 2019, 119, 5298.

[5] V. Beachley, X. Wen, Materials Science and Engineering: C 2009, 29, 663.

[6] J. M. Deitzel, J. Kleinmeyer, D. Harris, N. C. Beck Tan, Polymer 2001, 42, 261.

[7] S. H. Tan, R. Inai, M. Kotaki, S. Ramakrishna, Polymer 2005, 46, 6128.

[8] S. A. Theron, E. Zussman, A. L. Yarin, Polymer 2004, 45, 2017.

[9] H. Fashandi, M. Karimi, Polymer 2012, 53, 5832.

[10] C. Borgnakke, R. E. Sonntag, in Fundamentals of Thermodynamics, John Wiley \& Sons, 2013.

[11] J. Doshi, D. H. Reneker, Journal of Electrostatics 1995, 35, 151.

[12] S. Agarwal, J. H. Wendorff, A. Greiner, Polymer 2008, 49, 5603.

[13] V. Milleret, T. Hefti, H. Hall, V. Vogel, D. Eberli, Acta Biomaterialia 2012, 8, 4349.

[14] R. Wang, Y. Liu, B. Li, B. S. Hsiao, B. Chu, J. Membr. Sci. 2012, 392, 167.

[15] N. Tomczak, N. F. van Hulst, G. J. Vancso, Macromolecules 2005, 38, 7863.

[16] S. Somvipart, S. Kanokpanont, R. Rangkupan, J. Ratanavaraporn, S. Damrongsakkul, International Journal of Biological Macromolecules 2013, 55, 176.

[17] T. Li, X. Ding, L. Tian, J. Hu, X. Yang, S. Ramakrishna, Materials Science and Engineering: C 2017, 74, 471.

[18] N. Thakur, A. S. Ranganath, K. Agarwal, A. Baji, Macromolecular Materials and Engineering 2017, 302, 1700124.

[19] J. Lin, B. Ding, J. Yang, J. Yu, G. Sun, Nanoscale 2012, 4, 176.

[20] H.-S. Bae, A. Haider, K. M. K. Selim, D.-Y. Kang, E.-J. Kim, I.-K. Kang, J Polym Res 2013, 20, 158.

[21] M. Cheng, Z. Qin, S. Hu, H. Yu, M. Zhu, Cellulose 2017, 24, 219.

[22] W. Yang, F. Yang, Y. Wang, S. K. Both, J. A. Jansen, Acta Biomaterialia 2013, 9, 4505.

[23] S. Lee, S. Cho, M. Kim, G. Jin, U. Jeong, J.-H. Jang, ACS Appl. Mater. Interfaces 2014, 6, 1082.

[24] X. Li, Y. Zhang, H. Li, H. Chen, Y. Ding, W. Yang, Desalination 2014, 344, 266.

[25] B. B. Rothrauff, B. B. Lauro, G. Yang, R. E. Debski, V. Musahl, R. S. Tuan, Tissue Engineering Part A 2017, 23, 378.

[26] B. Ding, X. Wang, J. Yu, M. Wang, J. Mater. Chem. 2011, 21, 12784.

[27] P. K. Szewczyk, U. Stachewicz, Advances in Colloid and Interface Science 2020, 286, 102315.

[28] A. Greiner, J. H. Wendorff, in Self-Assembled Nanomaterials I (Ed: T. Shimizu), Springer Berlin Heidelberg, Berlin, Heidelberg, 2008, pp. 107-171.

[29] Y. Cai, M. Gevelber, J Mater Sci 2013, 48, 7812.

[30] A. L. Yarin, S. Koombhongse, D. H. Reneker, Journal of Applied Physics 2001, 89, 3018.

[31] Y. Cai, M. Gevelber, Journal of Materials Science 2017, 52, 2605.

[32] J. Pelipenko, J. Kristl, B. Janković, S. Baumgartner, P. Kocbek, International Journal of Pharmaceutics 2013, 456, 125.

[33] S. Tripatanasuwan, Z. Zhong, D. H. Reneker, Polymer 2007, 48, 5742.

[34] G. Collins, J. Federici, Y. Imura, L. H. Catalani, Journal of Applied Physics 2012, 111, 044701.

[35] M. Liang, A. Hébraud, G. Schlatter, Polymer 2020, 200, 122576.

[36] V. A. Mohnen, in Electrical Processes in Atmospheres, Steinkopff, 1976, pp. 1-17.

[37] I. Dogu, Textile Research Journal 1984, 54, 111.

[38] L. Huang, N.-N. Bui, S. S. Manickam, J. R. McCutcheon, J. Polym. Sci. B Polym. Phys. 2011, 49, 1734. 
[39] G.-T. Kim, J.-S. Lee, J.-H. Shin, Y.-C. Ahn, Y.-J. Hwang, H.-S. Shin, J.-K. Lee, C.-M. Sung, Korean J. Chem. Eng. 2005, 22, 783.

[40] O. Hardick, B. Stevens, D. G. Bracewell, J Mater Sci 2011, 46, 3890.

[41] R. M. Nezarati, M. B. Eifert, E. Cosgriff-Hernandez, Tissue Eng Part C Methods 2013, 19, 810.

[42] G. Yazgan, A. M. Popa, R. M. Rossi, K. Maniura-Weber, J. Puigmartí-Luis, D. Crespy, G. Fortunato, Polymer 2015, 66, 268.

[43] H. Fashandi, M. Karimi, Thermochimica Acta 2012, 547, 38.

[44] H. Fashandi, M. Karimi, Ind. Eng. Chem. Res. 2014, 53, 235.

[45] L. Natarajan, J. New, A. Dasari, S. Yu, M. A. Manan, $R S C A d v .2014,4,44082$.

[46] S. Megelski, J. S. Stephens, D. B. Chase, J. F. Rabolt, Macromolecules 2002, 35, 8456.

[47] P. Lu, Y. Xia, Langmuir 2013, 29, 7070.

[48] S. De Vrieze, T. V. Camp, A. Nelvig, B. Hagström, P. Westbroek, K. De Clerck, J Mater Sci 2008, 44, 1357.

[49] R. A. Basheer, A. R. Hopkins, P. G. Rasmussen, Macromolecules 1999, 32, 4706.

[50] G. J. Kettle, Polymer 1977, 18, 742.

[51] S. De Vrieze, B. De Schoenmaker, Ö. Ceylan, J. Depuydt, L. Van Landuyt, H. Rahier, G. Van Assche, K. De Clerck, J. Appl. Polym. Sci. 2011, 119, 2984.

[52] E. Marsano, L. Francis, F. Giunco, Journal of Applied Polymer Science 2010, 117, 1754.

[53] B. D. Schoenmaker, L. V. der Schueren, R. Zugle, A. Goethals, P. Westbroek, P. Kiekens, T. Nyokong, K. D. Clerck, J Mater Sci 2012, 48, 1746.

[54] I. İçoğlu, T. Oğulata, The Journal of The Textile Institute 2015, 106, 57.

[55] M. Yu, R.-H. Dong, X. Yan, G.-F. Yu, M.-H. You, X. Ning, Y.-Z. Long, Macromolecular Materials and Engineering 2017, 302, 1700002.

[56] International Journal of Nonlinear Sciences and Numerical Simulation 2007, 8, 393.

[57] D. Lukas, A. Sarkar, P. Pokorny, Journal of Applied Physics 2008, 103, 084309.

[58] J. Xiong, Y. Liu, A. Li, L. Wei, L. Wang, X. Qin, J. Yu, Materials \& Design 2021, 197, 109247.

[59] O. Jirsak, F. Sanetrnik, D. Lukas, V. Kotek, L. Martinova, J. Chaloupek, 2009, US Patent 7585437.

[60] H. Niu, T. Lin, X. Wang, Journal of Applied Polymer Science 2009, 114, 3524.

[61] S.-L. Liu, Y.-Y. Huang, H.-D. Zhang, B. Sun, J.-C. Zhang, Y.-Z. Long, Materials Research Innovations 2014, 18, S4.

[62] F. Yalcinkaya, Arabian Journal of Chemistry 2019, 12, 5162.

[63] X. Wang, B. Ding, G. Sun, M. Wang, J. Yu, Progress in Materials Science 2013, 58, 1173.

[64] J. Lin, F. Tian, Y. Shang, F. Wang, B. Ding, J. Yu, Nanoscale 2012, 4, 5316.

[65] G. Yazgan, R. I. Dmitriev, V. Tyagi, J. Jenkins, G.-M. Rotaru, M. Rottmar, R. M. Rossi, C. Toncelli, D. B. Papkovsky, K. Maniura-Weber, G. Fortunato, Scientific Reports 2017, 7, 158.

[66] C. L. Casper, J. S. Stephens, N. G. Tassi, D. B. Chase, J. F. Rabolt, Macromolecules 2004, 37, 573.

[67] E. S. Medeiros, L. H. C. Mattoso, R. D. Offeman, D. F. Wood, W. J. Orts, Can. J. Chem. 2008, 86, 590.

[68] J.-Y. Park, I.-H. Lee, Journal of Nanoscience and Nanotechnology 2010, 10, 3473.

[69] W. Liu, C. Huang, X. Jin, Nanoscale Res Lett 2014, 9, 1.

[70] J. Zheng, H. Zhang, Z. Zhao, C. C. Han, Polymer 2012, 53, 546.

[71] A. Bandegi, M. R. Moghbeli, J. Appl. Polym. Sci. 2018, 135, 45586.

[72] L. Tian, D. Ji, S. Zhang, X. He, S. Ramakrishna, Q. Zhang, Small 2020, 16, 2001743.

[73] A. L. Yarin, W. Kataphinan, D. H. Reneker, Journal of Applied Physics 2005, $98,064501$.

[74] B. Zaarour, L. Zhu, X. Jin, Polymers for Advanced Technologies 2020, 31, 2659.

[75] M. Halabi, M. Mann-Lahav, V. Beilin, G. E. Shter, O. Elishav, G. S. Grader, D. R. Dekel, Polymers 2020, 12, 1020.

[76] V. E. Kalayci, P. K. Patra, Y. K. Kim, S. C. Ugbolue, S. B. Warner, Polymer 2005, 46, 7191.

[77] Z. Tang, C. Qiu, J. R. McCutcheon, K. Yoon, H. Ma, D. Fang, E. Lee, C. Kopp, B. S. Hsiao, B. Chu, Journal of Polymer Science Part B: Polymer Physics 2009, 47, 2288.

[78] C. Thammawong, S. Buchatip, A. Petchsuk, P. Tangboriboonrat, N. Chanunpanich, M. Opaprakasit, P. Sreearunothai, P. Opaprakasit, Polym Eng Sci 2014, 54, 472. 
[79] C.-L. Pai, M. C. Boyce, G. C. Rutledge, Macromolecules 2009, 42, 2102.

[80] S. Yao, X. Wang, X. Liu, R. Wang, C. Deng, F. Cui, Journal of nanoscience and nanotechnology 2013, 13, 4752 .

[81] W. Zhao, B. Yalcin, M. Cakmak, Synthetic Metals 2015, 203, 107.

[82] S. Y. Bak, G. J. Yoon, S. W. Lee, H. W. Kim, Materials Letters 2016, 181, 136.

[83] F. H. Alshafei, D. A. Simonetti, Chemical Engineering Science 2020, 219, 115547.

[84] J. Wisniak, A. Tamir, Journal of Chemical and Engineering Data 1977, 22, 253.

[85] H. Batzer, U. Kreibich, Polymer Bulletin 1981, 5, 585.

[86] S. Yan, Y. Yu, R. Ma, J. Fang, Polymers for Advanced Technologies 2019, 30, 1635.

[87] M. O. Aijaz, M. R. Karim, H. F. Alharbi, N. H. Alharthi, Polymer 2019, 180, 121665.

[88] X. Ni, W. Cheng, S. Huan, D. Wang, G. Han, Carbohydrate Polymers 2019, 206, 29.

[89] R. Ghobeira, M. Asadian, C. Vercruysse, H. Declercq, N. De Geyter, R. Morent, Polymer 2018, $157,19$.

[90] M. Putti, M. Simonet, R. Solberg, G. W. M. Peters, Polymer 2015, 63, 189.

[91] Z. Wang, C. Zhao, Z. Pan, Journal of Colloid and Interface Science 2015, 441, 121.

[92] Z. Wang, Z. Pan, Applied Surface Science 2015, 356, 1168.

[93] H. Li, Z. Wang, H. Zhang, Z. Pan, Polymers 2018, 10, 1085.

[94] L. Pauchard, C. Allain, Europhys. Lett. 2003, 62, 897.

[95] M. Srinivasarao, D. Collings, A. Philips, S. Patel, Science 2001, 292, 79.

[96] M. Simsek, Journal of Materials Research 2020, 35, 332.

[97] B. Zaarour, L. Zhu, C. Huang, X. Jin, Nanoscale Research Letters 2018, 13, 285.

[98] L. Li, R. Li, M. Li, Z. Rong, T. Fang, RSC Adv. 2014, 4, 27914.

[99] B. Zaarour, L. Zhu, C. Huang, X. Jin, Journal of Applied Polymer Science 2019, 136, 47049.

[100] E. Rezabeigi, N. R. Demarquette, Macromolecular Rapid Communications 2019, 40, 1800880.

[101] D. H. Reneker, A. L. Yarin, H. Fong, S. Koombhongse, Journal of Applied Physics 2000, 87, 4531.

[102] C. Wan, C. R. Bowen, J. Mater. Chem. A 2017, 5, 3091.

[103] J. I. Kim, J. C. Lee, M. J. Kim, C. H. Park, C. S. Kim, Materials Letters 2019, 236, 510.

[104] T.-H. Kong, S.-S. Lee, G.-J. Choi, I.-K. Park, ACS Applied Materials \& Interfaces 2020, 12, 17836.

[105] P. K. Szewczyk, A. Gradys, S. K. Kim, L. Persano, M. Marzec, A. Kryshtal, T. Busolo, A. Toncelli, D. Pisignano, A. Bernasik, S. Kar-Narayan, P. Sajkiewicz, U. Stachewicz, ACS Applied Materials \& Interfaces 2020, 12, 13575.

[106] D. L. Chinaglia, R. Gregorio Jr., J. C. Stefanello, R. A. Pisani Altafim, W. Wirges, F. Wang, R. Gerhard, Journal of Applied Polymer Science 2010, 116, 785.

[107] P. Sajkiewicz, A. Wasiak, Z. Gocłowski, European Polymer Journal 1999, 35, 423.

[108] H. Yoshida, K. Sakuragi, Bulletin of the Chemical Society of Japan 2019, 92, 927.

[109] A. Celebioglu, T. Uyar, Chem. Commun. 2010, 46, 6903.

[110] A. Celebioglu, T. Uyar, Nanoscale 2012, 621.

[111] M. Oster, A. Hébraud, S. Gallet, A. Lapp, E. Pollet, L. Avérous, G. Schlatter, Macromol Rapid Commun 2015, 36, 292.

[112] M. Oster, G. Schlatter, S. Gallet, R. Baati, E. Pollet, C. Gaillard, L. Avérous, C. Fajolles, A. Hébraud, Journal of Materials Chemistry B 2017, 5, 2181.

[113] T. Kida, S. Sato, H. Yoshida, A. Teragaki, M. Akashi, Chem. Commun. 2014, 50, 14245.

[114] W. W. Li, X. H. Qin, Advanced Materials Research 2014, 941-944, 1225.

[115] E. S. Cozza, O. Monticelli, E. Marsano, P. Cebe, Polymer International 2013, 62, 41.

[116] T. Han, D. H. Reneker, A. L. Yarin, Polymer 2007, 48, 6064.

[117] B. Ding, C. Li, Y. Miyauchi, O. Kuwaki, S. Shiratori, Nanotechnology 2006, 17, 3685.

[118] T. Liang, M. Parhizkar, M. Edirisinghe, S. Mahalingam, European Polymer Journal 2014, 61, 72.

[119] Y. Yang, Z. Jia, Q. Li, Z. Guan, IEEE Transactions on Dielectrics and Electrical Insulation 2006, 13,580 .

[120] E. Schoolaert, P. Ryckx, J. Geltmeyer, S. Maji, P. H. M. Van Steenberge, D. R. D’hooge, R. Hoogenboom, K. De Clerck, ACS Appl. Mater. Interfaces 2017, 9, 24100. 
[121] D. Li, Y. Wang, Y. Xia, Advanced Materials 2004, 16, 361.

[122] D. Li, G. Ouyang, J. T. McCann, Y. Xia, Nano Lett. 2005, 5, 913.

[123] D. Zhang, J. Chang, Advanced Materials 2007, 19, 3664.

[124] N. Lavielle, A. Hébraud, C. Mendoza, A. Ferrand, N. Benkirane-Jessel, G. Schlatter, Macromol. Mater. Eng. 2012, 297, 958.

[125] C. R. Wittmer, A. Hébraud, S. Nedjari, G. Schlatter, Polymer 2014, 55, 5781.

[126] S. Nedjari, A. Hébraud, S. Eap, S. Siegwald, C. Mélart, N. Benkirane-Jessel, G. Schlatter, RSC Adv. 2015, 5, 83600.

[127] S. Nedjari, G. Schlatter, A. Hébraud, Materials Letters 2015, 142, 180.

[128] D. Li, G. Ouyang, J. T. McCann, Y. Xia, Nano Lett. 2005, 5, 913.

[129] D. Ahirwal, A. Hébraud, R. Kádár, M. Wilhelm, G. Schlatter, Soft Matter 2013, 9, 3164.

[130] X. Ye, X. Huang, Z. Xu, Chinese Journal of Polymer Science 2012, 30, 130.

[131] K. Chen, S. Zhang, B. Liu, X. Mao, G. Sun, J. Yu, S. S. Al-Deyab, B. Ding, RSC Adv. 2014, 4, 45760.

[132] X. Wang, Y. Si, X. Wang, J. Yang, B. Ding, L. Chen, Z. Hu, J. Yu, Nanoscale 2013, 5, 886.

[133] S. Zhang, H. Liu, X. Yin, Z. Li, J. Yu, B. Ding, Sci Rep 2017, 7, 40550.

[134] H. Okuzaki, T. Takahashi, N. Miyajima, Y. Suzuki, T. Kuwabara, Macromolecules 2006, 39, 4276.

[135] M. M. Li, Y. Z. Long, Materials Science Forum 2011, 688, 95.

[136] W. Su, N. Wu, B. Wang, H. Bao, Y. Wang, Materials Letters 2018, 216, 228.

[137] C. A. Bonino, K. Efimenko, S. I. Jeong, M. D. Krebs, E. Alsberg, S. A. Khan, Small 2012, 8, 1928.

[138] S. I. Jeong, N. A. Burns, C. A. Bonino, I. K. Kwon, S. A. Khan, E. Alsberg, J. Mater. Chem. B 2014, $2,8116$.

[139] J. W. Yoon, Y. Park, J. Kim, C. H. Park, Fashion and Textiles 2017, 4, 9.

[140] B. Sun, Y.-Z. Long, F. Yu, M.-M. Li, H.-D. Zhang, W.-J. Li, T.-X. Xu, Nanoscale 2012, 4, 2134.

[141] X. Wang, B. Ding, J. Yu, J. Yang, Colloids and Surfaces B: Biointerfaces 2011, 86, 345.

[142] B. K. Bhattacharjee, T. M. Schneider, M. P. Brenner, G. H. McKinley, G. C. Rutledge, J. Appl. Phys. 2010, 107, 044306.

[143] S. Zhang, K. Chen, J. Yu, B. Ding, Polymer 2015, 74, 182.

[144] J. Hu, X. Wang, B. Ding, J. Lin, J. Yu, G. Sun, Macromol. Rapid Commun. 2011, 32, 1729.

[145] H. Zhu, R. Li, X. Wu, K. Chen, J. Che, European Polymer Journal 2017, 86, 154.

[146] Y. Jiang, D. Fang, G. Song, J. Nie, B. Chen, G. Ma, New J. Chem. 2013, 37, 2917.

[147] G. Jiang, L. Luo, L. Tan, J. Wang, S. Zhang, F. Zhang, J. Jin, ACS Appl. Mater. Interfaces 2018, 10,28210 .

[148] M. Simonet, O. D. Schneider, P. Neuenschwander, W. J. Stark, Polymer Engineering \& Science 2007, 47, 2020.

[149] M. Simonet, N. Stingelin, J. G. F. Wismans, C. W. J. Oomens, A. Driessen-Mol, F. P. T. Baaijens, J. Mater. Chem. B 2014, 2, 305.

[150] M. F. Leong, M. Z. Rasheed, T. C. Lim, K. S. Chian, Journal of Biomedical Materials Research Part A 2009, 91A, 231.

[151] M. F. Leong, W. Y. Chan, K. S. Chian, M. Z. Rasheed, J. M. Anderson, Journal of Biomedical Materials Research Part A 2010, 94A, 1141.

[152] F. A. Sheikh, H. W. Ju, J. M. Lee, B. M. Moon, H. J. Park, O. J. Lee, J.-H. Kim, D.-K. Kim, C. H. Park, Nanomedicine: Nanotechnology, Biology and Medicine 2015, 11, 681.

[153] L. Huang, J. Huang, H. Shao, X. Hu, C. Cao, S. Fan, L. Song, Y. Zhang, Materials Science and Engineering: C 2019, 94, 179.

[154] M. F. Leong, W. Y. Chan, K. S. Chian, Nanomedicine 2013, 8, 555.

[155] N. Lavielle, A. Hébraud, G. Schlatter, L. Thöny-Meyer, R. M. Rossi, A.-M. Popa, ACS Appl. Mater. Interfaces 2013, 5, 10090.

[156] W. Choi, G. H. Kim, J. H. Shin, G. Lim, T. An, Nanoscale Research Letters 2017, 12, 610.

[157] M. M. Trexler, C. Hoffman, D. A. Smith, T. J. Montalbano, M. P. Yeager, D. Trigg, S. Nimer, X. Calderón-Colón, C. Peitsch, Z. Xia, Journal of Polymer Science Part B: Polymer Physics 2019, 57, 563. 
[158] B. Barua, M. C. Saha, Polymer Engineering \& Science 2018, 58, 998.

[159] P. K. Szewczyk, D. P. Ura, U. Stachewicz, Fibers 2020, 8, DOI 10.3390/fib8100065.

[160] F. Flaig, H. Ragot, A. Simon, G. Revet, M. Kitsara, L. Kitasato, A. Hébraud, O. Agbulut, G. Schlatter, ACS Biomater. Sci. Eng. 2020, 6, 2388.

[161] T. G. Kim, H. J. Chung, T. G. Park, Acta Biomaterialia 2008, 4, 1611.

[162] E. Norris, C. Ramos-Rivera, G. Poologasundarampillai, J. P. Clark, Q. Ju, A. Obata, J. Hanna V., T. Kasuga, C. A. Mitchell, G. Jell, J. R. Jones, Biomedical Materials 2020, 15, 015014.

[163] O. D. Schneider, F. Weber, T. J. Brunner, S. Loher, M. Ehrbar, P. R. Schmidlin, W. J. Stark, Acta Biomaterialia 2009, 5, 1775.

[164] F. A. Formica, E. Öztürk, S. C. Hess, W. J. Stark, K. Maniura-Weber, M. Rottmar, M. ZenobiWong, Advanced Healthcare Materials 2016, 5, 3129.

[165] A. A. Bulysheva, G. L. Bowlin, A. J. Klingelhutz, W. A. Yeudall, Journal of Biomedical Materials Research Part A 2012, 100A, 757.

[166] S. Hwang, W. Kim, H. Yoon, S. Jeon, ACS Sens. 2017, 2, 1355.

[167] W. Kim, E. Park, S. Jeon, Sensors (Switzerland) 2020, $20,437$.

[168] X. Wang, J. Wang, Y. Si, B. Ding, J. Yu, G. Sun, W. Luo, G. Zheng, Nanoscale 2012, 4, 7585.

[169] X. Wang, B. Ding, J. Yu, Y. Si, S. Yang, G. Sun, Nanoscale 2011, 3, 911.

[170] Y. Wen, Y. Li, Y. Si, X. Wang, F. Li, J. Yu, B. Ding, Talanta 2015, 144, 1146.

[171] J. Zhang, L. Liu, Y. Si, J. Yu, B. Ding, Mater. Chem. Front. 2021, 5, 97.

[172] I. Sas, R. E. Gorga, J. A. Joines, K. A. Thoney, Journal of Polymer Science Part B: Polymer Physics 2012, 50, 824.

[173] D. Ceylan, S. Dogu, B. Karacik, S. D. Yakan, O. S. Okay, O. Okay, Environ. Sci. Technol. 2009, $43,3846$.

[174] Y. Tang, Z. Liu, K. Zhao, Journal of Applied Polymer Science 2019, 136, 47262.

[175] I. E. Paulus, T. Moss, A. Greiner, Macromolecular Materials and Engineering 2016, 301, 1225.

[176] Y. Li, F. Yang, J. Yu, B. Ding, Advanced Materials Interfaces 2016, 3, 1600516.

[177] Y. Liu, C. Duong-Viet, J. Luo, A. Hébraud, G. Schlatter, O. Ersen, J.-M. Nhut, C. Pham-Huu, Chem CatChem 2015, 7, 2957.

[178] Y. Liu, J. Luo, C. Helleu, M. Behr, H. Ba, T. Romero, A. Hébraud, G. Schlatter, O. Ersen, D. S. $\mathrm{Su}$, C. Pham-Huu, J. Mater. Chem. A 2017, 5, 2151.

[179] M.-H. Sun, S.-Z. Huang, L.-H. Chen, Y. Li, X.-Y. Yang, Z.-Y. Yuan, B.-L. Su, Chem. Soc. Rev. 2016, 45, 3479.

[180] Y. Liu, J. Luo, Y. Shin, S. Moldovan, O. Ersen, A. Hébraud, G. Schlatter, C. Pham-Huu, C. Meny, Nature Communications 2016, 7, 11532.

[181] P. Aghasiloo, M. Yousefzadeh, M. Latifi, R. Jose, Journal of Alloys and Compounds 2019, 790, 257.

[182] C. A. Pope, D. W. Dockery, Journal of the Air \& Waste Management Association 2006, 56, 709.

[183] S. Wang, X. Zhao, X. Yin, J. Yu, B. Ding, ACS Appl. Mater. Interfaces 2016, 8, 23985.

[184] R.-R. Cai, S.-Z. Li, L.-Z. Zhang, Y. Lei, Science of the Total Environment 2020, 725, 138297.

[185] H. Liu, S. Zhang, L. Liu, J. Yu, B. Ding, Advanced Functional Materials 2019, 29, 1904108.

[186] J. Song, Z. Li, H. Wu, ACS Appl. Mater. Interfaces 2020, 12, 33447. 